\title{
Longitudinal associations between functional disability, depression, and suicide in middle-aged and older adults
}

Julie Ann Lutz

West Virginia University, jalutz@mix.wvu.edu

Follow this and additional works at: https://researchrepository.wvu.edu/etd

Part of the Clinical Psychology Commons, and the Geropsychology Commons

\section{Recommended Citation}

Lutz, Julie Ann, "Longitudinal associations between functional disability, depression, and suicide in middle-aged and older adults" (2019). Graduate Theses, Dissertations, and Problem Reports. 4122. https://researchrepository.wvu.edu/etd/4122

This Dissertation is protected by copyright and/or related rights. It has been brought to you by the The Research Repository @ WVU with permission from the rights-holder(s). You are free to use this Dissertation in any way that is permitted by the copyright and related rights legislation that applies to your use. For other uses you must obtain permission from the rights-holder(s) directly, unless additional rights are indicated by a Creative Commons license in the record and/ or on the work itself. This Dissertation has been accepted for inclusion in WVU Graduate Theses, Dissertations, and Problem Reports collection by an authorized administrator of The Research Repository @ WVU.

For more information, please contact researchrepository@mail.wvu.edu. 
Longitudinal associations between functional disability, depression, and suicide in middle-aged and older adults

$$
\text { Julie Lutz }
$$

\author{
Doctoral Dissertation submitted \\ to the Eberly College of Arts and Sciences \\ at West Virginia University \\ in partial fulfillment of the requirements for the degree of \\ Doctor of Philosophy in \\ Clinical Psychology
}

\begin{abstract}
Amy Fiske, Ph.D., Chair
Barry Edelstein, Ph.D.

Nicholas Turiano, Ph.D.

Ian Rockett, Ph.D.

Chandra Reynolds, Ph.D.

Department of Psychology
\end{abstract}

\author{
Morgantown, West Virginia \\ 2018
}

Keywords: functional disability, depressive symptoms, suicide, middle age, older adults Copyright 2018 Julie Lutz 


\begin{abstract}
Longitudinal associations between functional disability, depression, and suicide in middle-aged and older adults
\end{abstract}

Julie Lutz

Suicide is a significant public health problem among older adults in the United States and around the world. There has been little research to date on longitudinal change in functional disability and depressive symptoms, both of which have been posited to be associated with suicide risk among middle-aged and older adults, and their association with death by suicide. The aims of this study were to 1) characterize longitudinal change in functional disability and depressive symptoms separately; 2) characterize the directional associations between functional disability and depressive symptoms in bivariate models; and 3) determine how different patterns of change among these variables are associated with risk for death by suicide. The study used two larger population-based longitudinal datasets, the Health and Retirement Study (HRS) in the United States, and the Swedish Twin Registry (STR). Results from HRS indicated that change in functional disability and depressive symptoms is characterized by a complex system of effects, including both systematic, linear change effects and proportional change based on the level of each variable at each wave. Functional disability generally increased across time and depressive symptoms remained relatively stable, with some slight increases. A bivariate model where level of disability was associated with subsequent change in depressive symptoms was supported by the data, and latent class membership representing group differences in patterns of change within this model predicted risk for death by suicide. Cognitive impairment was significantly associated with lower risk for death by suicide, and appeared to have a suppressor effect on the association of class membership with death by suicide. In STR, functional disability did not predict risk for death by suicide. Further research is needed to characterize the complex longitudinal associations between disability and depression and their effects on suicide risk, taking into account cognitive status. 


\section{Table of Contents}

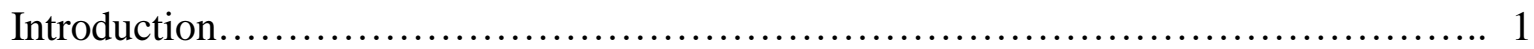

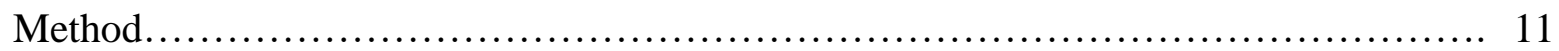

Results.................................................................... 21

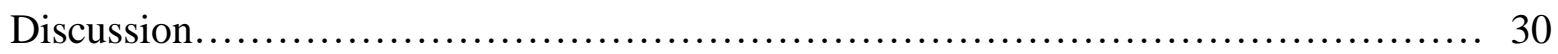

References......................................................... 40

Tables.................................................................. 47

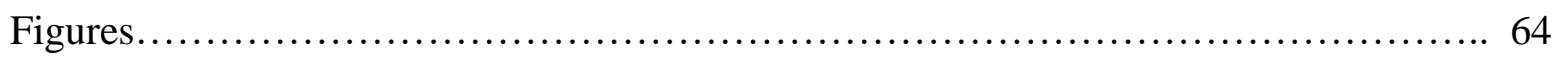

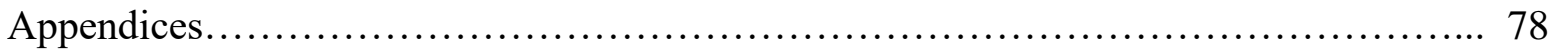


Longitudinal associations between functional disability, depression, and suicide in middle-aged and older adults

Suicide among middle-aged and older adults is a critical public health problem in the United States and around the globe. Middle-aged and older adults have the highest suicide rates in the U.S., with rates of 16.7 per 100,000 adults age 65 and over and 19.5 per 100,000 adults age 50 to 64 in 2016, in comparison to the national average rate of 13.4 per 100,000 in 2016 (Centers for Disease Control and Prevention [CDC], 2018). Older men drive these high suicide rates in the U.S., with rates as high as 29.6 and 31.0 in 50-64 year-olds and 65-84 year-olds, respectively. Among men, suicide rates skyrocket with increasing age, such that men age 85 and older have rates as high as 48.0 suicides for every 100,000 individuals - more than three times the average suicide rate in the general population. Similar patterns by age and gender are exhibited in many regions around the world, with older men (age 70 and older) yielding the highest suicide rates (World Health Organization [WHO], 2014).

Given such concerning rates of suicide among the middle-aged and older population, it has become crucial to answer questions about what factors contribute to risk for suicide within this population. Currently, though more and more research is being disseminated regarding suicide in middle-aged and older adults, this population is still frequently underrepresented in the suicide literature (Szanto et al., 2013), particularly given the fact that this population is continually growing due to the Baby Boomer cohort reaching older adulthood. Some general information on individual risk factors for suicide is available (e.g., see review by Conwell, Van Orden, \& Caine, 2011). However, it is necessary to develop and test specific models of the mechanisms through which such risk factors may increase suicide risk in middle age and older 
adulthood. The proposed study will test a model of how disability and depression may increase risk of suicide in this population.

\section{Disability and suicide}

Disability is a highly prevalent health-related concern in middle age and late life, and this prevalence consistently increases with age. The Americans With Disabilities Act (1990) defines disability as "a physical or mental impairment that substantially limits one or more major life activities" of an individual. The prevalence of any disability in 45-54 year-olds and 55-64 yearolds was $19.7 \%$ and $28.7 \%$, respectively, in the United States in 2010 (Brault, 2012). Though the prevalence of severe disability was not as high in these age groups, it still impacted a large proportion of the population (13.8\% and $20.4 \%$ for $45-54$ and 55-64 year-olds, respectively). These numbers only increase with each successive age group. In 2008-2012, more than onethird of all adults age 65 and older were impacted by at least one disability (He \& Larsen, 2014). More than $70 \%$ of those age 85 and older had at least one disability, with the majority impacted by multiple disabilities. According to the World Health Organization (2002), the term "disability" is an overarching term under which several constructs can be subsumed; it may include limitations in completing daily activities. For the purposes of this study, functional disability is operationalized as the presence and level of limitation or impairment of daily life activities. Functional disability can impact a variety of areas of life, and by definition pervades into an individual's everyday functioning. Therefore, it is arguable that functional disability could serve as a major risk factor for a variety of negative psychosocial outcomes, including directly impacting suicide-related behavior and/or indirectly impacting the risk factors for suicide. 
Research to date has been somewhat mixed regarding the associations between functional disability and a variety of suicide-related behaviors, including suicidal ideation, suicide attempts, and death by suicide, in middle-aged and older adults. The majority of research on these associations in this age group has focused on suicidal ideation and used cross-sectional designs (Lutz \& Fiske, 2017). The majority of those studies show an association between greater levels of functional disability and suicidal ideation. Only a handful of studies have examined the association between functional disability and death by suicide. The majority of these utilized psychological autopsy designs, which depend upon the retrospective report of proxy informants for individuals who died by suicide. Two psychological autopsy studies without comparison groups found that large proportions of individuals who died by suicide had functional and/or physical limitations. Filiberti and colleagues (2001) found that four of five cancer patients who died by suicide had experienced functional and/or physical impairment. Préville, Boyer, Hébert, Bravo, and Seguin (2005a) found that approximately half of the older adults in their sample who had died by suicide had moderate or severe functional limitations at the time of their death. Twenty-two percent had experienced a decline in level of functioning over the 6 months prior to their death.

Psychological autopsy studies that incorporated comparison groups have been more informative regarding the association between functional disability and death by suicide. Four studies utilized living control subjects. These studies all found that the presence of, or greater levels of, functional disability was associated with being in the suicide case groups, rather than control groups (Conwell et al., 2000; 2010; Rubenowitz, Waern, Wilhelmson, \& Allebeck, 2001; Tsoh et al., 2005). One study that utilized control subjects that had died by natural causes, however, found conflicting results. In this study, moderate or severe functional impairment was 
more prevalent in the control group than in the suicide case group, and the control group experienced greater decline in functioning, on average, over the 6 months prior to death (Préville, Hébert, Boyer, Bravo, \& Seguin, 2005b).

The psychological autopsy studies discussed above have several limitations. First, the dependence of psychological autopsy studies on retrospective, proxy reports leaves them vulnerable to possible misreporting and error. Some of the proxy interviews in these studies occurred several years after the suicide of the individual. For example, interviews occurred two to eight years after the suicide in the study by Filiberti and colleagues (2001). Another important consideration for psychological autopsy studies is the choice of control group. The majority of the studies with control groups utilized living controls, which are generally selected from the healthiest portions of the population (e.g., independent community-dwelling older adults) and therefore may not be completely representative of the entire older-adult population (Préville et al., 2005b). In addition, the difference in information obtained through proxy report for suicide decedents and self-report for living control subjects (i.e., different methods) may introduce bias (Isometsä, 2001). However, the use of a control group consisting of individuals who died by natural causes may be likely to introduce bias in the opposite direction of using living controls. Those who die by natural causes are likely to have more health problems and greater levels of impairment (Préville et al., 2005b). Because of these limitations in psychological autopsy studies, it is important to conduct more research using prospective designs, where self-report of functioning and other risk factors can be obtained, and researchers can follow up to determine which participants died by suicide over a period of time. Such designs, however, require enormous quantities of data on very large samples, due to the low base rate of death by suicide. 
Only one study to date used prospective data to examine the association between functional disability and death by suicide in older adults. The researchers had self-report data on functional disability from over 14,000 individuals, and followed up over a ten-year period, during which 21 individuals had died by suicide (Turvey et al., 2002). There was no difference in level of functioning at baseline between those who later died by suicide during the following ten years, or those who died by other means or were still living after ten years. However, there was no additional follow-up or data collection between the baseline and death outcome within the following ten years, which limits these findings. Level of functional disability (and other risk factors for suicide) could change multiple times in the course of ten years, therefore obscuring any effect of disability on suicide years later. It is instead necessary to continually assess changes in functional disability in a prospective design that includes multiple times of measurement and incorporates temporal associations between such onset or exacerbation of functional disability and risk of suicide. Though there are some inconsistent results regarding the association between functional disability and suicide in the studies described above, the majority of findings suggest that greater levels of disability are associated with increased risk for suicide.

\section{Association between functional disability and depression}

Depression is one of the most prominent risk factors for suicide (Conwell, Duberstein, \& Caine, 2002; Conwell et al., 2011), and functional disability may affect risk of suicide through its effects on depression. There is a bidirectional association between functional disability and depression, such that disability is predictive of depression and depression is associated with worsened functional impairment (Lebowitz et al., 1997). Some studies examining the association between functional disability and suicide-related behavior have thus incorporated 
depression in their analyses. In a systematic review of the literature, most studies examining the association between disability and suicidal ideation in middle-aged and older adults found that including depression diagnosis or level of depressive symptoms as a covariate led to decreased association between functional disability and suicidal ideation (Lutz \& Fiske, 2017). In some the association became nonsignificant, but in the majority of studies depression did not fully account for the association between disability and suicidal ideation. Two studies directly assessed depression as a mediator in this association in older adults, and found significant results (Ahn \& Kim, 2015; Park et al., 2014). Regarding the association between functional disability and death by suicide, Conwell and colleagues (2000) performed comparisons of depressed living control subjects and depressed suicide decedents. They found that almost no indices of functional impairment significantly differed between groups, though they had previously differed when the comparison group was all (including non-depressed) living controls. Consistent with these findings, Conwell, Duberstein, and Crane (2002) conclude, in a review of the risk factors for suicide in older adults, that though physical health and functional disability serve as risk factors for suicide, this association tends to decline after controlling for depression and related problems. They posit that risk is mostly or completely mediated by affective disorders. Though the findings of these studies point to a mediational role for depression in the association between disability and suicide, more formal analyses, particularly using longitudinal data to parse out temporal associations, are necessary to confirm this.

Taking into account the research to date on the associations between functional disability, suicide, and depression, a model is proposed where the onset of, or increase in, functional disability in later life leads to increased risk of suicide through subsequent onset or increase in depressive symptoms. Examining such models of the trajectories that lead to increased risk of 
suicide is an important next step in the progress of the field of suicide prevention in older adults.

Finding the mechanisms through which functional disability may increase risk of suicide allows us to determine the most appropriate avenues for prevention and intervention efforts. To confidently determine the temporal nature of these associations and establish the trajectory leading to increased risk of suicide, longitudinal data with multiple times of measurement are necessary (Singer \& Willett, 2003).

\section{Trajectories of change in depression in late life}

Several studies have examined the longitudinal trajectory of changes in depressive symptoms in late life. Chui and colleagues (2015) examined age-related trajectories of depressive symptoms in late life. Depressive symptoms increased with age. This rate of increase was greater among older men, compared to women, such that women in early older adulthood reported greater depressive symptoms than men, and men in late older adulthood reported greater depressive symptoms than women. The increase in depressive symptoms with age interacts with cohort effects. With longitudinal data, Yang (2007) found an overall pattern of increased depressive symptoms with age, similar to other studies. However, cohort was also associated with differences in age-related trajectories of depressive symptoms. There was an age-by-cohort interaction such that each cohort generally had higher levels of depression, but the within-cohort trajectory of symptom levels was positive (i.e., increasing) in later cohorts and negative (i.e., decreasing) in earlier cohorts. These findings show that growth (or decline) in depressive symptoms over time is linked with both age and cohort.

In a two-year longitudinal study examining trajectories of depressive symptoms in older adults, six trajectories emerged (Cui, Lyness, Tang, Tu, \& Conwell, 2008). Those without significant depressive symptoms at the beginning of the study either maintained a similar level of 
depressive symptoms or worsened to subsyndromal levels of depressive symptoms over time.

Those who began the study with subsyndromal or minor levels of depressive symptoms either remained unchanged, worsened, or improved over time. Finally, those with levels of depressive symptoms indicative of major depression at the beginning of the study maintained a similar level of symptoms over time.

A couple of studies have examined associations between trajectories of depression and disability in later life. Fauth, Gerstorf, Ram, and Malmberg (2014) examined whether trajectories of depressive symptoms in late life were better characterized by chronological age, time to and from onset of disability, or time to death. They found that time to and from disability and time to death were better models for the trajectories of depressive symptoms than chronological age, with time to death yielding the best model fit. This suggests that disability and mortality processes are more predictive of depressive symptom trajectories than age. In regard to disability in particular, depressive symptoms were generally highest immediately after onset of disability, with the lowest levels of depressive symptoms at times more distal from the onset of disability (Fauth et al., 2014). Lenze and colleagues (2005) studied the associations between 4-year longitudinal trajectories of depression and disability among older adults. Individuals who were persistently depressed during the study period experienced a greater increase in disability over that time, compared to those who were temporarily depressed or never depressed during the study period. Though these studies point to the association between growth in depression and disability in late life, the direction of these associations remains unclear.

\section{Cognitive status and suicide risk}

Research on the associations between dementia/Alzheimer's disease and suicide risk indicate that, though suicide risk may increase in the early stages of dementia and just after 
diagnosis, risk is relatively low in later stages of dementia (e.g. Serafini et al., 2016). Haw, Harwood, and Hawton (2009) reviewed the literature on dementia and suicide and concluded that mild cognitive impairment may be associated with higher suicide rates, but that suicide rates were generally lower among those with dementia. Cognitive status could affect risk of suicide on a variety of fronts. In early stages of cognitive impairment, an individual may have greater insight into their impairment, and may be more likely to become depressed and hopeless and more likely to exhibit suicidal behavior (Serafini et al., 2016). In later stages, however, more severe dementia may impair an individual's capability to plan a suicide attempt and to lethally harm oneself. Therefore, in considering the associations of depression and disability with suicide risk, it is important to examine their interaction with cognitive status. Cognitive impairment may be a main contributing factor to functional disability, and may also be associated with particular patterns of depression and suicidality.

\section{Statement of the problem and hypotheses}

Little is currently known about the trajectories that increase risk for death by suicide in later life. Research has suggested that one risk factor in middle-aged and older adults may be increasing levels of functional disability. Functional disability may impact a number of areas of life that influence risk of suicide. The proposed model posits that functional disability leads to the onset of or increase in depressive symptoms. As depression has been established as one of the most significant risk factors for suicide, this serves as a major mechanism through which functional disability may increase risk of suicide. This study examined this model in large population-based data sets in the United States and Sweden. The aims and hypotheses were as follows. 


\section{Aim 1: Estimate longitudinal change in functional disability and depressive}

symptoms separately. Longitudinal growth models were estimated to examine patterns of change in functional disability and in depressive symptoms. I hypothesized that, in the sample as a whole, functional disability would generally increase with age (i.e. across waves), consistent with the greater levels of disability with age in the population. I hypothesized that there would be variability in growth trajectories for depressive symptoms, similar to those found by Cui and colleagues (2008), but generally depressive symptoms would increase across age, consistent with findings by Chui and colleagues (2015) and Yang (2007).

\section{Aim 2: Examine temporal precedence in the longitudinal associations between} functional disability and depressive symptoms. Estimations of paths between the growth models for functional disability and depressive symptoms that were described in Aim 1 were examined to determine whether changes in functional disability would temporally precede changes in depressive symptoms, and whether there was a bidirectional association between disability and depressive symptoms. If there was a bidirectional association, these paths were to be examined to determine whether this association would be stronger in one direction. I hypothesized that there would be a bidirectional association between functional disability and depressive symptoms, and that this association would be somewhat stronger with functional disability preceding increases in depressive symptoms, based in part on the work of Fauth and colleagues (2014) that showed trajectories of depressive symptoms could be characterized by time from disability onset.

\section{Aim 3: Test the veracity of a bivariate longitudinal model of functional disability} and depressive symptoms in predicting hazard for suicide. Latent class growth analysis and growth mixture modeling were used to examine how groups with different patterns of change in 
functional disability and depressive symptoms would differ in hazard for late-life suicide. I hypothesized that there would be latent classes representing different trajectories of functional disability and depressive symptoms. Additionally, I hypothesized that high levels of functional disability and depressive symptoms would be associated with greater hazard for suicide, based on past research on the associations between disability, depression, and risk of suicide.

However, analyses regarding the nature of the trajectories of functional disability and depressive symptoms (e.g. increasing, fluctuating, stable) and their associations with suicide were exploratory.

Interactions of latent class membership with cognitive status in predicting hazard for suicide were also assessed. I hypothesized that significant cognitive impairment (great enough impairment to lead to a diagnosis of dementia or prevent an individual from completing an interview) would be associated with generally lower risk of suicide, consistent with findings reviewed by Haw, Harwood, and Hawton (2009) and Serafini and colleagues (2016). It was anticipated that this might manifest such that the risk of suicide would be buffered among individuals with cognitive impairment in latent classes that would otherwise be associated with greater risk of suicide.

\section{Method}

The current study utilized data from two large population-based studies with linkages to death index data - the Health and Retirement Study (HRS) and the Swedish Twin Registry (STR). The HRS and STR are excellent sources of detailed, longitudinal psychosocial and health information on large population-based samples merged with cause of death information. Both data sets were used due to differences in measurement of variables involved in the study and to increase confidence in findings through partial replication. 


\section{Sample 1 - Health and Retirement Study (HRS)}

Participants and procedures. The HRS is a population-based study in the United States. Data are collected prospectively every two years, and initial data collection began in 1992. Waves from 1994 to 2014 were used in this study. The HRS includes data from a total of 35,222 individuals over the age of 50 years who participated in at least one of the waves included in this study. Data consist of interviews and questionnaires. The HRS data are merged with data from the National Death Index (NDI), the national cause of death file, so that date and cause of death can be determined for deceased individuals in the sample.

Measures. The HRS includes measures of functional disability, depressive symptoms, and cause and date of death. See Table 2 for a summary of missing data for the variables described below.

Functional disability. Data from the 1994 data collection to 2014 were used. Interview questions inquired whether the individual had any difficulty with 12 physical activities, 5 activities of daily living (ADLs), and 5 instrumental activities of daily living (IADLs). Responses on items asking about difficulty performing individual activities were indicated as “yes" (to experiencing difficulty), “no," "can’t do [activity]," “don't do [activity]”, or missing (due to refusal to answer or other reasons). Responses of "yes" or "can't do" were counted as difficulty/disability in that activity, whereas responses of “don't do" were counted as missing. However, in sensitivity analyses, responses of “don't do" were counted as difficulty/disability in that activity. Two groups of physical activity items were grouped together, as they measure variations of the same type of activity such that, if an individual indicated they had no difficulty with the most strenuous activity (e.g. running), they were not asked about "easier" versions of the activity (see Appendix). Therefore, when scoring, each individual had scores for 9 physical 
activities. ADLs were measured only among participants who indicated they had at least one difficulty with physical activities. Participants who indicated no difficulty with physical activities were assigned scores of 0 (no disability) for all ADLs. IADLs were measured in all participants. Total scores were calculated from the number (out of 19) of activities for which the individual was reported to have difficulty. Measures of ADLs and IADLs, with totals of the numbers of activities a person either cannot perform or needs assistance in performing, are commonly used as measures of level of functional disability (see Spector \& Fleishman, 1998). Because total scores across all three categories of activities are not frequently used, the internal consistency of this 19-item total was tested in the most recent wave (2014). In this wave, the alpha coefficient was .90 , suggesting good internal consistency when all physical activity, ADL, and IADL items were combined into one measure.

In the 1994 wave, which includes part of the sample for Wave 2 (called the AHEAD sample/cohort), one IADL was not measured (meal preparation). For this wave, the total score was prorated, such that the mean of all other items was calculated, then multiplied by 19 . For all waves, missingness on functional activities was prorated in the same way if participants completed at least 14 out of 19 items (approximately $75 \%$ ).

Depressive symptoms. The HRS data include scores on a shortened form of the Center for Epidemiological Studies Depression scale (CESD) from 1994 to 2014. This form of the CESD contains 8 items, chosen from the original 20-item measure based on factor analysis (Steffick, 2000). HRS participants responded "yes" or "no" regarding whether they experienced these 8 symptoms "much of the time during the past week": "you felt depressed," "you felt that everything you did was an effort," "your sleep was restless," "you were happy," "you 
felt lonely," "you enjoyed life," "you felt sad," "you could not get going." Items worded positively (e.g. "you were happy") are reverse-scored, and items are scored "0" for no and "1" for yes. Total scores range from 0 to 8 (Steffick, 2000). This version of the CESD has exhibited good internal reliability ( $\alpha$ ranges from .77 to .83 in multiple samples and waves of HRS; Steffick, 2000). In all waves, missingness was prorated among participants who completed at least 6 out of 8 items (75\%), such that the mean of all completed items was calculated, then multiplied by 8 .

Suicide. National Death Index (NDI) codes for suicide (E950-E959 [ICD-9] and X60X84 and Y87.0 [ICD-10]) and deaths of undetermined intent (E980-E989 [ICD-9] and Y10-Y34, Y87.2, and Y89.9 [ICD-10]) were used for indicating death by suicide. The HRS data are merged every 2 or 3 years with NDI codes for cause of death. The most current available merge occurred in 2013 and includes data through the end of 2011 and earlier. Deaths that are coded in the NDI as suicides or undetermined deaths were considered as deaths by suicide for the proposed analyses. This is consistent with previous research methods in this field. Deaths of undetermined intent are conventionally combined with suicide deaths in research studies due to the evidence that a majority of deaths classified as "undetermined intent" are suicides (e.g., Rockett, Kapusta, \& Bhandari, 2011). In fact, some countries, such as the United Kingdom, define suicide by both suicide codes and undetermined intent codes (e.g., UK Department of Health, 2014). In addition, inclusion of only deaths coded specifically as suicides could lead to underrepresentation of certain demographic groups, such as racial/ethnic minorities and women, whose deaths are less likely to be categorized as suicides (Rockett et al., 2010). Analyses were conducted with only deaths coded as suicides and then again with both suicides and undetermined deaths to examine similarities or differences that might have occured when the two 
methods were compared. In addition, deaths coded as due to drug intoxication (E850-E858 [ICD-9] and X40-X44 [ICD-10]) were included as suicides in a sensitivity analysis, based on theory defining death due to drug intoxication as a form of self-harm or self-destructive behavior (Rockett et al., 2016; Rockett, Caine, Connery, \& Greenfield, 2018; Rockett, Kapusta, \& Coben, 2014; Rockett, Smith, et al., 2014; Stone et al., 2017).

Cognitive status. A dichotomous score on cognitive status was calculated based on the most recent wave of data available for each individual (either prior to death, prior to 2011, or at the last wave in which they participated). The score was based on the individual's proxy status and reason for proxy (i.e. indication that the individual's proxy responded in that wave due to cognitive limitations of the participant), and/or report of a diagnosis of memory problems, dementia or Alzheimer's disease by a provider (see Appendix A for wording of items). Assessment of these variables varied by wave. Appendix A indicates what variables were available in each wave. Coding for cognitive status was completed based on the available variables in each wave. If the individual had a proxy due to cognitive limitations at the most recent wave, or indicated at the most recent wave that they had previously been diagnosed with dementia or Alzheimer's disease, they were coded as cognitively impaired. If they indicated they had no proxy or proxy was not due to cognitive limitations, and were not diagnosed with memory problems, they were coded as not cognitively impaired. If responses on any available variables were missing, they were coded as missing on cognitive status for the current analyses.

\section{Sample 2 - Swedish Twin Registry (STR)}

Participants and procedures. The STR is a population-based twin database in Sweden. It is the one of the largest twin registries in the world, and data have been collected since the early 1960s via various cross-sectional and longitudinal studies. The Screening Across the 
Lifespan Twin study (SALT) data were used for the purpose of this study. SALT consisted of a comprehensive telephone interview - including questions regarding physical health, mental health, and social variables - administered between 1998 and 2002 to all living twins born prior to 1958 in the registry (Lichtenstein et al., 2006). Activities of daily living were assessed only among individuals age 65 and older in SALT - therefore the current study includes only older adults in this age range. The STR is also linked periodically with the Swedish Cause of Death Register so that cause of death can be determined for the individuals in the sample. Cause of death data were available for deaths occurring through the end of 2012.

Measures. The STR includes measures of functional disability, depression, cause and date of death, and zygosity of twins.

Functional disability. In SALT, interviewees were asked "Do you receive any help, for example with...?" and were prompted with five ADLs or IADLs, including: "Buying or preparing food," "Personal care (for example dressing, bathing)," "Remembering to take your medicine," "Housework," and "Handling your finances." There was also an "Other [activity]" option, a "No" option, a "Don't know" option, and an option to refuse to answer the question. A total number of the activities for which the individual responded that they received help serves as a measure of disability. Responses of “don't know" or refusal to answer were coded as missing.

Depressive symptoms. The SALT interview included an 11-item shortened version of the CESD (Kohout, Berkman, Evans, \& Cornoni-Huntley, 1993). The 11 items include 7 of the ones listed above that were included in the HRS (except for "you had a lot of energy"), as well as "You did not feel like eating. Your appetite was poor," "People were unfriendly," and "You felt that people disliked you." SALT retained the original CESD response format, which is a frequency format, rather than a dichotomous "yes" or "no." Responses could be "Never or 
almost never," "Seldom," "Often," or "Always or almost always," as well as the option of refusing to respond. Items are scored 0 to 3, with "Never or almost never" scored as 0 and “Always or almost always" scored as 3. Positively-worded items are reverse-scored, and total scores range from 0 to 33. The 11-item version of the CESD has adequate internal reliability ( $\alpha$ $=.76$ ) among adults age 65 and older, and the pattern of factor loadings of the items in a factor analysis is consistent with that of the original 20-item scale (Kohout et al., 1993).

Suicide. Deaths were coded by ICD versions 6 through 10. The same ICD codes as defined above for the HRS NDI file, and equivalents for versions 8 and earlier (E950-E959 [ICD-8], E963 \& E970-E979 [ICD-7 \& ICD-6] for suicides; E980-E989 [ICD-8] for deaths of undetermined intent - no similar codes in ICD-6 or -7; E850-E859 [ICD-8], E870-E878 [ICD-7 \& ICD-6] for deaths by drug intoxication), were used to categorize deaths by suicide in the STR data.

\section{Analyses}

Aim 1. To test the longitudinal patterns of change in functional disability and depressive symptoms in HRS, separate univariate dual change score models (DCSM) were estimated (see Jajodia, 2012). These models were partially modeled on those presented by Sbarra and Allen (2009). As shown in Figure 1, the two DCSMs included latent (i.e., "true score") variables representing levels of disability or depressive symptoms at each measurement time. These latent variables were estimated from the measured HRS variables (i.e., CESD scores, ADL scores) at each time point. The latent scores at each time point were then used to estimate change in disability or depressive symptoms between each measurement time, proportional to the level of the variable at the prior wave. In other words, one's level of functional disability at wave 1 , for example, predicts the extent to which disability changes between waves 1 and 2 . If this 
parameter is significant and positive, then higher levels of disability at wave 1 are associated with greater increase between waves 1 and 2. Growth indices (i.e., slope and intercept) were added to characterize systematic linear change over time. Sex and age at first wave were included as time-invariant predictors. For the purposes of these analyses, data were organized such that all participants are entered in "Time 1" (i.e. their first wave of participation) and subsequent waves in order, as opposed to by year of wave. For example, if Participant A began participation in 1994 and completed 5 subsequent waves in order (i.e. through 2004) and Participant B began participation in 2000 and completed the next wave (2002) and a wave in 2006 (skipping 2004), then Participant A has data entered at Times 1 through 5 and Participant B has data entered at Times 1 through 2 and Time 4. In addition, all DCSMs (including the bivariate models discussed below) account for clustering within the same household. Inclusion of both systematic, or stable, growth parameters as well as auto-proportional parameters in these DCSM models combines time series analysis (e.g. auto-regressive and cross-lag models) with growth curve analysis (Jajodia, 2012). Therefore, models were used because they are capable of addressing the complex questions of examination of change or growth over time within each variable, as well as cross-lagged temporal associations between changes or growth in multiple variables across waves (as described in Aim 2).

Aim 2. To examine temporal precedence in the associations between functional disability and depressive symptoms in HRS, a bivariate DCSM including both disability and depressive symptoms was estimated (see Jajodia, 2012). Again, the script was partially modeled from Sbarra and Allen (2009). As shown in Figure 2, the model was run while allowing different paths representing associations between disability and depressive symptoms to be estimated. The strength of these temporal associations were assessed by comparing the fit of 
models estimating 1) no paths between level of either variable and subsequent change in the other; 2) paths from disability level at a given time of measurement to change in depressive symptoms from that measurement to the next (paths marked "a" in Figure 2); 3) paths from depressive symptom level at a given time of measurement to change in disability from that measurement to the next (paths marked " $b$ " in Figure 2); and 4) paths in both directions (both paths "a" and "b" in Figure 2). As in Aim 1, sex and age at first wave were included as timeinvariant predictors.

A final model was selected based on comparison of a variety of fit indices across models (i.e., Akaike information criterion [AIC], Bayesian information criterion [BIC], root mean square error of approximation [RMSEA], comparative fit index [CFI], Tucker-Lewis index [TLI], and standardized root mean square residual [SRMR]) and theoretical rationale. The selected model was used in subsequent analyses.

Aim 3. The associations between longitudinal change in functional disability and depressive symptoms and hazard for suicide were examined using latent class analysis (LCA), incorporating the bivariate DCSM described above and depicted in Figure 2, and survival analysis. Whereas the models previously described provided indices for change among the entire HRS sample, the aim of these analyses was to identify sub-groups with different patterns of change, and examine whether these different trajectories predicted different hazard rates for suicide.

First, the number and characteristics of underlying latent classes were determined using LCA with the bivariate DCSM selected from Aim 2. Using LCA allowed for the initial identification of different classes of growth trajectories (see Jung \& Wickrama, 2008). The final number of classes was selected based on a combination of fit indices (e.g., Bayesian information 
criterion [BIC]; Lo, Mendell, \& Rubin likelihood ratio test [LMR-LRT]; and entropy) and theoretical rationale (Jung \& Wickrama, 2008).

Once the latent classes were identified, individual probability of belonging to a given class was estimated. The full three-step method for auxiliary variables outlined by Asparouhov and Muthén (2014) was not implemented in this study due to computational demands of a joint LCA-survival model, but served as a guide for the analytical approach. Probabilities of membership in each class were used to predict the survival/hazard model for death by suicide. Due to issues of multicollinearity, only the probabilities of two classes were included as independent variables. Along with latent class probabilities, cognitive status was included as a moderator, such that its interactions with the probabilities for membership in each latent class in predicting hazard for suicide were assessed. The survival model was conducted using Poisson regression. In HRS, only month and date of entry into the HRS study and birthdate were available; therefore, these dates in the survival model were set at the first day of the given month. Month, year, and date were available with NDI data for individuals who had died by the end of 2011, and the end-of-study date was set at December 31, 2011 for all participants who were not deceased. The survival model accounted for household clustering.

Though longitudinal growth and associations between functional disability and depressive symptoms could not be examined in the STR data, due to the availability of only one time of measurement, the association between disability and depressive symptoms at one time point and hazard for suicide over time could be modelled. Poisson regression models predicting hazard for death by suicide, first with just level of disability (controlling for sex and age), and then with both disability and depressive symptoms, at the time of the SALT interview as timeinvariant predictors, were estimated (e.g., see Singer \& Willett, 2003, chapter 14). For a 
comparison of the fit of models including only ADLs versus those including both ADLs and depressive symptoms, the quasi-likelihood under the independence model criterion (QIC) was examined - smaller values represent better fit. This analysis provided additional information regarding the associations between disability, depressive symptoms, and suicide in a separate sample from HRS, allowing for some examination of the generalizability of findings.

\section{Results}

\section{Sample 1 - Health and Retirement study}

Sample characteristics. Among 35,222 participants included in this study, any individuals with some complete data on the variables involved in a particular DCSM (either univariate or bivariate) were included in that DCSM, utilizing maximum likelihood estimation for missing data. In total, 34,901 had complete enough data to be included in the bivariate DCSMs and latent class analysis. The number of individuals who participated in each wave varied, as did the total number of waves of data available for each individual. See Table 1 for a summary of the number of waves of data available for participants in HRS. See Table 2 for the number of participants involved in each wave of HRS, as well as descriptive information on the major study variables (depressive symptoms and functional limitations). Fifty-six percent of participants were female. Age at entry to the study ranged from 50 to 106 years.

Data for ADLs were somewhat skewed and kurtotic (ADL skew ranged from 1.19 to 1.47 across waves, kurtosis ranged from 1.02 to 2.59 across waves; CESD skew ranged from 1.09 to 1.35 , kurtosis ranged from 0.55 to 1.47$)$. Therefore, transformation of both variables was attempted. Transformations did not affect the level of skew and kurtosis of CESD data, so original data were used in the analyses. A square-root transformation slightly reduced skew and kurtosis of ADL data. All DCSM analyses were run twice - with original ADL data and with 
transformed ADL data. Results were not appreciably different. Therefore, for ease of interpretation, results using the original ADL data are reported.

Aim 1 - Univariate DCSMs. See Table 3 for results of the univariate DCSMs for depressive symptoms and functional limitations (i.e., ADLs). Both models resulted in good fit indices (RMSEA below .05, CFI above .95, TLI above .95, and SRMR below .08), suggesting the resultant models characterize change in functional limitations and depressive symptoms well.

The DCSMs yield both systematic, linear change parameters (the slope for the variable) and auto-proportional change parameters (the association between level of the variable at one time point and the amount and direction of change from that time point to the next). The univariate model for functional limitations resulted in systematic change parameters indicating a negative average slope for functional limitations over time (i.e. decrease in ADL impairment across time), but a positive association between level of ADLs at one time point and change in ADLs from that time point to the next. This proportional change also accumulates across time points. Therefore, the overall change in ADLs is positive over time, as the proportional change has the effect of increasing scores. Additionally, a significant negative association between ADL intercept and slope suggests that the scores of individuals starting with greater levels of impairment tend to have more negative systematic change in impairment across time than those of individuals starting with lower levels of impairment. This association is tempered, however, by the opposing effect of the proportional change. Change is also associated with age and sex, such that male sex is associated positively with systematic change in ADLs (i.e., systematic/linear change in males' ADLs is more positive), and greater age is associated positively with systematic change (i.e., systematic/linear change in ADLs is more positive with increasing age). 
The univariate model for depressive symptoms resulted in a positive average slope for change in depressive symptoms over time (i.e., increase in depressive symptoms across time), and a positive association between intercept and slope (i.e. those with higher levels of depressive symptoms have greater increase in depressive symptoms over time) but a negative proportional association between level of CESD at one time point and change from that time point to the next (i.e. greater levels of depressive symptoms at one time point are associated with less increase in depressive symptoms from that time point to the next). This suggests a dampening effect of the proportional change on the systematic, linear change at higher levels of depressive symptoms. Age and sex are also associated with change in depressive symptoms, such that male sex is associated with less increase in CESD scores over time, and increased age is associated with greater increase in CESD scores over time.

Aim 2 - Bivariate DCSM. See Table 4 for results of the bivariate DCSMs testing the associations between functional limitations and depressive symptoms, as well as directionality of these associations, over time. The models with no directional proportional paths between depressive symptoms and functional limitations, with functional limitations predicting change in depressive symptoms, and with depressive symptoms predicting change in functional limitations, yielded good fit indices. Note in Figure 2 that the static growth/change parameters were allowed to covary between both variables in all models, including the one with no directional paths between functional limitations and depressive symptoms, so some association could be indicated without the presence of the directional paths. The bidirectional model including both pathways between functional limitations and depressive symptoms did not converge; therefore, no results are reported. 
The three completed models were compared based on fit indices and theoretical rationale to select an appropriate model as the basis for the subsequent latent class and survival analysis. Fit indices were somewhat comparable across all three models. The chi-square difference test used to examine the significance of the difference in loglikelihood values (i.e., -2LL) for each of the models. The two unidirectional models were compared to the model with no paths between functional limitations and depressive symptoms. The chi-square difference test was significant for both models, suggesting they each better characterized change in functional limitations and depressive symptoms compared to the model with no directional paths between the two variables.

Due to the similarity in fit indices between the two unidirectional models, graphs of the results were consulted to visually examine the patterns of change and associations between functional limitations and depressive symptoms indicated by the results. In addition, theoretical rationale was used to select a final model. Figures 3 and 4 show plots of change over time in ADLs and depressive symptoms, as well as vector plots of the association between ADLs and depressive symptoms, for the ADL-to-CESD and CESD-to-ADL models, respectively. Patterns indicated in Figure 3, of the ADL-to-CESD model, as well as the individual parameters (shown in Table 4) appear more consistent with known associations between increased depressive symptoms and greater functional limitations, and with the change patterns found in the univariate models. Therefore, the ADL-to-CESD model was selected for further analyses.

In the ADL-to-CESD model, change patterns for ADLs and CESD scores separately were consistent with the results of each of the univariate models. The association between ADLs at one time point and change in CESD scores from that time point to the next was positive and significant, suggesting that greater functional impairment is associated with additional positive 
change, or less negative change, in depressive symptoms subsequently. As shown in Figure 3c (within the ellipse that indicates the area with the most observed scores), at lower CESD scores and lower ADL scores, the effect of ADLs on CESD scores is not very consistent. However, at greater levels of ADL impairment, change in CESD scores stabilizes and becomes generally flat or less pronounced. As ADL impairment increases, CESD scores appear to stabilize around a moderate level rather than increasing or decreasing drastically. Also, at lower levels of depressive symptoms, there appears to be more positive change in symptoms occurring at higher ADL values, while at higher CESD and ADL levels there appears to be more negative change in CESD.

Sensitivity analyses were completed with ADLs coded somewhat differently, such that responses of “don't do" were coded as impairment on a given activity. Results of all univariate and bivariate models with these recoded ADLs were nearly identical to results with the original coding. Therefore, only results with the original coding (“don't do" responses coded as missing) are reported here.

Aim 3 - Latent classes \& suicide risk. Latent class analyses were run, using the ADLto-CESD bivariate DCSM, with two, three, and four classes. Table 5 shows fit indices for the three latent class analyses. The best loglikelihood value was not replicated in the four-class model, and thus one cannot be confident that the results do not represent a local solution. Therefore, the results of the four-class model should not be interpreted. The three-class solution fit the data better than the two-class solution, yielding a smaller BIC and greater entropy index. The classes were composed of approximately $16 \%, 74 \%$, and $10 \%$ of participants.

Table 6 shows the parameters of the DCSM model for each of the three classes, and Figures 5, 6, and 7 show plots of change over time in ADLs and depressive symptoms and vector 
plots of the association between ADLs and depressive symptoms, for each of the three classes. The first class (16\%) is characterized by higher average intercepts for ADLs and depressive symptoms. The first class also shows a greater systematic, linear decrease in ADL limitations over time (as indicated by a more negative average slope) and a smaller positive autoproportional effect of ADL levels in preceding waves on ADL change to the next wave. Figures 5a and $b$ show patterns of change in CESD and ADL scores; depressive symptoms among this class generally start at higher levels and as a group appear to remain stable, whereas ADLs also start at higher levels and exhibit more variety between individuals in change patterns (including individuals increasing and decreasing in ADL impairment over time) than the other classes. Also, in the first class, there is a negative proportional association between ADL impairment level at preceding stages and change in depressive symptoms by the subsequent waves (i.e. greater levels of impairment are associated with less subsequent growth in depressive symptoms). Altogether, as shown in Figure 5c, at lower levels of depressive symptoms and across all levels of functional impairment, depressive symptoms increase as functional impairment changes in any direction. However, around CESD scores of 4 or 5 out of 8 , depressive symptoms become stable. At higher levels of depressive symptoms, scores are decreasing across time.

The second class $(74 \%)$ is characterized by somewhat lower intercepts for ADL limitations and depressive symptoms, and a less negative systematic slope effect in ADLs. Figures 6a and b show that levels of depressive symptoms start within the lower range and remain relatively stable, whereas ADL impairment starts in the lower range and generally shows stability or large increases over time. In this class, the effect of level of ADL impairment in preceding waves and change in depressive symptoms by subsequent waves is positive (i.e. 
greater levels of functional impairment are associated with subsequently more positive change in depressive symptoms). As shown in Figure 6c, at lower levels of ADL impairment and CESD scores, change in CESD scores converges toward a relatively low level (around 2 or 3 out of 8), such that when scores are 2 or below they are increasing, and when they are 3 or higher they are decreasing. At moderate levels of ADL impairment, change in CESD scores increase from scores of 3 or below and decreases from scores of 4 or greater. Overall, change in CESD scores appears to converge toward moderate levels, though the level at which change in CESD scores converges increases somewhat as ADL impairment increases.

Finally, in the third class (10\%), intercepts for ADLs and CESD scores are moderate (between those in the first and second classes). Both the positive linear, systematic growth in CESD scores and the negative auto-proportional effect are of a somewhat smaller magnitude. Meanwhile, the positive auto-proportional effect of preceding ADL levels on subsequent ADL change is of greater magnitude in this class. Figures $7 \mathrm{a}$ and $\mathrm{b}$ show that CESD symptoms generally begin at a moderate level and remain stable or increase over time, whereas ADL impairment begins at a low to moderate level and shows more drastic increases over time. The effect of preceding ADL levels on subsequent change in depressive symptoms is positive, but smaller than the effect in the second class. Examining Figure 7c, it appears that, at lower levels of depressive symptoms and ADL impairment, CESD scores increase toward a moderate level, but at higher levels of depressive symptoms at the same level of ADL impairment, CESD scores decrease toward a moderate level. At higher levels of ADL impairment, lower CESD scores are increasing, but higher CESD scores remain quite stable.

Approximately $10 \%(n=3,431)$ of participants that had been included in the DCSMs and latent class analyses were missing on additional variables necessary for the survival analyses 
(i.e., cognitive status, birth date, or date of first interview in HRS). Therefore, the survival analyses had a sample size of 31,470 . Among those, there were 30 cases of death by suicide, 3 cases of death by undetermined intent, and 8 cases of death by drug intoxication through 2011 (see codes used under Measures). Approximately $8.7 \%$ of those involved in the survival analysis (16.0\% of Class $1,3.7 \%$ of Class 2 , and $32.0 \%$ of Class 3 ) were coded as having cognitive impairment in the last available data based on the criteria described in the method. Suicide rates within age bands were calculated. Poisson regressions were conducted with 1) suicides alone as outcome; 2) suicides and deaths of undetermined intent as outcome; and 3) suicides, undetermined, and overdose deaths as outcome. Models controlled for sex and age and were first run with probabilities for membership in classes 1 and 3 as independent variables, then with cognitive status at last available time point added, then with interactions between each class membership probability and cognitive status added. However, the models including the interactions yielded unstable results, as evidenced by very large confidence intervals and susceptibility to sporadic results, even after standardizing variables to control for multicollinearity. This may have resulted from low power due to the small number of suicide deaths. Therefore, only results of the first two models are reported, using the original forms of the variables rather than standardized versions, for ease of interpretation. See Table 7 for results. Incidence rate ratios (IRRs) and their confidence intervals do not account for household clustering, whereas chi-square score statistics do account for clustering.

Greater probability of Class 1 membership was consistently associated with greater risk for death by suicide, across all operationalizations (suicides only; suicides and undetermined deaths; and suicides, undetermined, and drug intoxication deaths). The incidence rate ratios (IRRs) indicate that $100 \%$ probability of membership in Class 1 is associated with approximately 
four to five times greater rates of death by suicide compared to $0 \%$ probability of membership in Class 1, though $95 \%$ confidence intervals are fairly broad due to the low overall rate of deaths by suicide. When cognitive status was included as a covariate, it was also consistently significantly associated with suicide risk, such that cognitive impairment is associated with only about 10$15 \%$ as much risk for suicide compared to those who are not cognitively impaired. Also, when cognitive status was included, probability of Class 3 membership approached significance. Male sex was also significantly associated with a three- to four-fold greater risk of death by suicide, but older age was not associated with risk.

Among participants included in the survival analysis, cognitive impairment at last available data collection was associated with greater ADL impairment at last available data collection (Satterthwaite $t$ for unequal variances $(\mathrm{df}=2662.9)=-78.49, p<.001$; cognitive impairment $M=12.36, \mathrm{SD}=5.08$; no cognitive impairment $M=4.03, \mathrm{SD}=3.91$ ). As a supplemental analysis, the Poisson models with age, sex, and class were re-run excluding all participants who had cognitive impairment $(n=2,694$ excluded, $n=28,776$ included in analysis). See Table 8 for the results. In these analyses, probability of Class 1 membership and probability of Class 3 membership were marginally significantly predictive of death by suicide.

\section{Sample 2 - Swedish Twin Registry}

Sample characteristics. Among 20,199 individuals age 65 and older who completed SALT interviews, 12,452 (62\%) completed ADLs. Of those, 359 (2.8\%) did not complete the CESD. In total, 12,093 individuals were included in the survival analyses. These included 18 cases of death by suicide, 4 cases of death by undetermined intent, and 2 deaths by drug intoxication. Forty-five percent of participants were male. 
Aim 3 - Suicide risk. Poisson regressions were conducted with 1) suicides alone as outcome; 2) suicides and deaths of undetermined intent as outcome; and 3) suicides, undetermined, and drug intoxication deaths as outcome. Models controlled for sex and age and were first run with ADLs as independent variable, then with both ADLs and depressive symptoms. Suicide rates within age bands were also calculated. See Table 9 for results.

Rates of suicide were highest among the oldest-old, with rates ranging from 30.4 to 45.6 per 100,000 people, depending on inclusion of deaths of undetermined intent and deaths by drug intoxication in the outcome. Among 65- to 74-year-olds rates ranged from 7.3 to 12.2 per 100,000, and among 75 to 84 year-olds rates ranged from 13.5 to 15.0 per 100,000. Models including both ADLs and depressive symptoms as predictors yielded slightly poorer fit (i.e. larger QIC values) compared to the models without depressive symptoms. However, fit was comparable and the models with ADLs and depressive symptoms showed a marginal or significant positive association between depressive symptoms and suicide deaths, such that every additional depressive symptom endorsed in SALT is associated with approximately $12 \%$ higher risk for death by suicide. In both models, and across operationalizations of the outcome (i.e. inclusion of undetermined and drug intoxication deaths), ADLs were not a significant predictor of suicide deaths. Male sex was significantly associated with greater risk of suicide.

\section{Discussion}

The aims of the current study were to 1) estimate patterns of longitudinal change in functional disability and depressive symptoms separately, 2) examine temporal precedence in longitudinal associations between functional disability and depressive symptoms, and 3) examine how a bivariate longitudinal model of functional disability and depressive symptoms can be informative for predicting risk for suicide. The Health and Retirement Study was used to carry 
out all three aims, while the Swedish Twin Registry was used to partially replicate the third aim, examining how functional disability and depressive symptoms at one time point are predictive of risk for suicide prospectively.

The univariate and bivariate dual change score models are best interpreted with consideration of all the parameters in combination, rather than individual parameters. Change in functional impairment or depressive symptoms is characterized both by a systematic, linear effect (the slope) and an auto-proportional feedback effect (the associations between each level of a variable at each given time point and change to the next time point; see Sbarra \& Allen, 2009, and Jajodia, 2009, for further explanation of these mechanisms). The combination of both defines the overall change in each variable. The systematic and proportional change effects may differ in direction (e.g., for ADLs, systematic change was negative but proportional change was positive). Therefore, plots of the change over time in each of the variables and of the association between changes in both variables are most informative in determining the overall nature of the change in impairment and depressive symptoms.

I hypothesized that functional disability would increase with time. Results of the models generally supported this hypothesis. Though the linear, systematic change in ADLs was negative, the proportional change was positive and plots of change in ADLs across time (e.g. Figure 3a) generally indicate an overall positive change, indicating that the proportional change from each individual time point exerts a greater effect on change over time than the systematic effect. However, there is some variation in ADL change patterns by age, sex, and, in later latent class analyses, most likely latent class.

I also hypothesized that there would be variability in change patterns in depressive symptoms, but that an increase over time in depressive symptoms would be most likely. 
Depressive symptoms were characterized by positive systematic change slopes but negative proportional change, which appeared to dampen the positive change effect. In general, levels of depressive symptoms appeared to remain relatively stable over time, with some having slight increases over time (e.g. see Figure 3b). Again, there was some variation in CESD change patterns by age, sex, and most likely latent class.

In regard to the directionality of the association between change in functional disability and depressive symptoms, I hypothesized that there would be a bidirectional association but that the effect of preceding ADL limitations on subsequent change in depressive symptoms would be stronger. The complete bidirectional DCSM did not converge despite attempting several variations, and therefore a direct comparison could not be conducted. The failure to converge may indicate that a model does not fit the data well. In any case, the two unidirectional bivariate models were compared to assess which fit the data best and was consistent with theoretical rationale. The unidirectional ADL to CESD model, where preceding levels of ADLs predict subsequent change in CESD scores but not vice versa, was selected as the model that was most consistent with theory, past research, and the results of the more simple models (i.e. the univariate DCSMs and the bivariate model without directional paths). Though both the ADL to CESD and the CESD to ADL models had quite similar fit indices (with the CESD to ADL model actually yielding slightly better fit indices), the results of the CESD to ADL model were not as consistent with the patterns of change indicated by the univariate and no-path bivariate models.

The association between functional disability and change in depressive symptoms, as illustrated in Figure 3c, suggests that, past a low-to-moderate level of functional disability, change in depressive symptoms tends to flatten or stabilize more and more with higher levels of disability. Therefore, though levels of disability are somewhat predictive of change in 
depressive symptoms at lower levels of disability, generally higher levels of disability do not appear to be associated with overall increases in depressive symptoms and instead appear to keep depressive symptoms at a more steady state.

Thus the unidirectional model with level of functional disability predicting subsequent change in depressive symptoms was used as the basis for latent class analysis to identify subgroups with differing patterns of change. Two- and three-class models were run, as well as a four-class model that was unable to be replicated and for which the solution could therefore not be interpreted with confidence. Between the two- and three-class models, the three-class one yielded better fit indices. Classes included approximately 16\%, 74\%, and 10\% of the sample. Probability of membership in each of these three classes was then used as a predictor of risk for death by suicide in a Poisson regression. The first class was consistently significantly associated with greater risk for death by suicide. This first class was characterized by overall higher levels of initial ADL impairment and depressive symptoms (see Table 6 and Figures 5a and b). In addition, there was variability in change in ADLs over time, with some decreasing in impairment levels and some increasing. Levels of depressive symptoms were generally stable around a moderate to high level. The results of these analyses are consistent with my hypothesis that overall higher levels of functional disability and depressive symptoms would be associated with risk for suicide. Interestingly, as shown in Figure 5c, level of depressive symptoms appears to converge toward a moderate level. It is also important to note that the majority of data (i.e., the ellipse in Figure 5c) fall within moderate CESD scores. The overall pattern of change in depressive symptoms to stabilize toward a moderate level may suggest some contribution of a regression-to-the-mean effect. The results also imply that decreasing ADL impairment would 
not necessarily have a straightforward effect on reduction in depressive symptoms, as depressive symptoms also converge toward a moderate level at lower ADL impairment as well.

When cognitive impairment was included in the Poisson regression model, it was significantly associated with lower risk for suicide. This is consistent with past research indicating that cognitive impairment is associated with reduced risk for death by suicide (e.g., Haw et al., 2009; Serafini et al., 2016). Interestingly, when cognitive impairment was included as a covariate, the third class approached a significant association with risk for death by suicide. This indicates that, when accounting for the presence of cognitive impairment, membership in Class 3 may also become predictive of greater suicide risk. Class 3 was characterized by initially moderate levels of depressive symptoms increasing over time and initially low to moderate functional impairment increasing rather drastically over time. The results of the analysis including cognitive impairment may point to a trend between this pattern of change and higher risk of suicide that is masked by higher prevalence of cognitive impairment (approximately $32 \%$ of this class had cognitive impairment, which is associated with lower risk of death by suicide) i.e., a suppressor effect. The results of models including interactions of cognitive status and class membership probability were unstable, and therefore a direct examination of an interaction between cognitive status and Class 3 in predicting suicide death could not be conducted.

The supplemental HRS survival analysis results excluding those with cognitive impairment yielded marginal or significant results for both Class 1 and Class 3 in predicting greater risk for suicide. These results tentatively support the interpretation that the greater level of cognitive impairment present in Class 3 masked the association between that pattern of change in functional disability and depressive symptoms and death by suicide. A limitation of these analyses, possibly contributing to the marginal significance of the results, is the low power due 
to the already low rate of deaths by suicide and the reduction in sample size by excluding those with cognitive impairment. More research examining these associations and interactions with greater power (in terms of number of suicide deaths) is necessary.

The results of the Health and Retirement Study analyses confirmed that generally higher levels of functional disability and depressive symptoms, jointly, are associated with death by suicide. However, not all individuals in Class 1 had increasing disability over time - there was variability in change in disability, and less drastic increases in disability than in the other classes. This may have, in part, been related to a type of ceiling effect, as ADL impairment began at higher levels in this group. But it shows that recent drastic increases in functional disability are not a necessary precursor for death by suicide, though more stable moderate to high levels of disability may be associated with suicide. Further, depressive symptoms were not drastically increasing in this group; they were remaining stable or decreasing slightly. Therefore, recent change may be less relevant to suicide risk than an overall elevated level of symptoms. The marginal-to-significant results regarding the Class 3 pattern in predicting death by suicide after accounting for cognitive impairment suggests that a more drastic increase in functional disability and depressive symptoms increasing steadily from moderate levels, if they occur in the absence of cognitive impairment, may also be associated with greater risk of death by suicide. However, cognitive impairment itself is more common in this group, and is associated with greater ADL impairment.

In the Swedish Twin Registry, activities of daily living were not significantly associated with risk for death by suicide in the Poisson regression survival model. Other studies on functional impairment and suicide have also failed to find a significant association, such as past psychological autopsy studies comparing suicide decedents with decedents of natural causes (e.g. 
Préville et al., 2005b). The STR results are consistent with the results found by Turvey et al. (2002), in a study with a similar design, where level of disability at one time point was not predictive of death by suicide over the next 20 years. The results of this analysis are not consistent with the higher overall levels of disability among Class 1, which was associated with greater risk of death by suicide, in the Health and Retirement Study. However, the HRS analyses allowed a more sophisticated approach of examining trajectories of change in disability over time and the utilization of repeated measures, sometimes up until fairly recently before death. A limitation of the STR analyses is that disability was measured only once in this study, therefore not allowing for an analysis of change in disability over time or an examination of levels of disability immediately preceding death. In addition, the STR analyses did not account for cognitive impairment which, as shown in the results of the HRS analyses, could mask associations of other variables with death by suicide. In future research, it will be important to account for cognitive status when examining the association between disability and death by suicide.

Also in the Swedish Twin Registry analyses, depressive symptoms were marginally associated with greater risk of death by suicide. In addition, in this sample, male sex was associated with greater risk of death by suicide, and later age trended toward greater risk for suicide, which is consistent with worldwide patterns of suicide rates (WHO, 2014).

Finally, in both HRS and STR, results of sensitivity analyses were relatively consistent across different outcome operationalizations (i.e., suicides only; suicides and undetermined deaths; and suicides, undetermined, and drug intoxication deaths). This suggests that these outcomes result from similar phenomenology, supporting the concept that deaths coded as due to 
undetermined intent and deaths coded as due to drug intoxication are likely to be inclusive of suicidal or self-destructive behavior (e.g., Rockett et al., 2011; 2014).

\section{Limitations}

This study is not without limitations. One of the main limitations is relatively low power for analyses involving suicide deaths, due to a small number of deaths by suicide in both the HRS and STR SALT samples. This was evident by the unstable results when attempting to assess the interaction between cognitive status and probability of class membership in HRS. This is a common limitation in research on death by suicide, due to the low base rate of the outcome and the person years necessary in a prospective study for an adequate sample of deaths by suicide. Further research would benefit from larger prospective data pools with ongoing death record linkages.

Another limitation of the study is data missingness. This was mainly problematic for the survival portion of the analyses, as this required complete data. Approximately $10 \%$ of HRS participants were missing data that were necessary for the Poisson regression, and were therefore excluded from analyses. A large portion of the STR SALT sample was missing data on ADLs and therefore could not be included in the survival analysis. The use of secondary data sets, while beneficial in terms of obtaining prospective data on large samples to link to suicide deaths, is subject to the data collection methods used in the original data collection. Maximum likelihood estimation was used for the DCSMs to mitigate the effects of missing data in those analyses.

Another limitation of this study was the use of shortened versions of the CESD. The use of dichotomous, rather than frequency-based, responses in the version used in HRS may limit the amount of variation in the severity of depressive symptoms in that sample, in comparison to the 
original frequency-based responses of the original CESD. Further, with fewer CESD items in both HRS and STR, some symptoms may not be represented by these measures.

The models used for HRS estimated change over time while controlling for age at first wave (i.e. essentially holding age to be the same). These models could be underpredicting the associations among these dynamics, as they may change with age. An even more precise method may estimate different models in different age groups, or use another method to incorporate these age-related dynamics in a way that is more nuanced than controlling for age at first wave. However, such a method would require greater power, particularly when estimating risk for suicide.

This study did not capture the onset of disability in the whole sample, therefore limiting interpretations about trajectories from the onset of disability. This is particularly evident in latent class 1 , as this class was characterized by somewhat higher levels of disability at the initial wave. Therefore, one cannot make any inferences as to the associations between disability, depressive symptoms, and suicidality closer to onset of disability.

\section{Conclusions and Future Directions}

This study confirmed that a model where level of disability is associated with subsequent change in depressive symptoms fits data from a population-based study well. However, the nature of that association is not as straightforward as a constant increase in depressive symptoms resulting from higher levels of disability. Instead, the association depends upon the current level of disability and depressive symptoms at any given time. Therefore, future clinical and research work should attend more to an individual's pattern of change and coping with disability and depression over time, rather than depending on a one-time measurement of risk factors. The results of the analyses described here suggest that functional disability and depressive symptoms 
that are more consistently at higher levels across time tend to be associated with greater risk of death by suicide. Middle-aged and older adults who present with higher, unrelenting levels of disability and depression should be assessed for suicidality. Interventions to reduce suicide risk among individuals with chronic disability and chronic depressive symptoms could also be impactful in this population. Depressive symptoms show a more distinct pattern of change associated with death by suicide than functional disability. Simple level of disability is not identified as a significant predictor of risk for death by suicide when measured at only one time point, as in the STR analyses. These results point to the necessity for a more nuanced and longitudinal view of risk factors for death by suicide than using single-time point, simple measures. More research is needed to replicate the findings from this study and to further elaborate on the longitudinal associations between disability, depression, and suicide in other samples and populations. In particular, research continuing to parse out the directionality and nature of associations between changes in disability and depressive symptoms, examining further subgroups with patterns of change that are more or less predictive of risk for suicide, and assessing interactions with cognitive impairment is needed. In further studies examining the association between disability and death by suicide, it is necessary to account for cognitive status, as greater ADL impairment is associated with greater likelihood of cognitive impairment, and cognitive impairment is associated with lower risk for death by suicide. Associations between functional limitations and suicide that are present in those without cognitive impairment may become masked in analyses that include those with cognitive impairment. The elements contributing to risk for suicide among middle-aged and older adults exhibit complex patterns of change over time, as well as complex associations among each other, and research is just beginning to characterize these influences. 


\section{References}

Ahmedani, B. K., Simon, G. E., Stewart, C., Beck, A., Waitzfelder, B. E., Rossom, R., ..., \& Solberg, L. I. (2014). Health care contacts in the year before suicide death. Journal of General Internal Medicine, 29, 870-877. doi: 10.1007/s11606-014-2767-3

Ahn, J., \& Kim, B. J. (2015). The relationships between functional limitation, depression, suicidal ideation, and coping in older Korean immigrants. Journal of Immigrant and Minority Health, 17, 1643-1653. doi: 10.1007/s10903-015-0204-2

American Psychiatric Association. (2013). Diagnostic and statistical manual of mental disorders (5th ed.). Washington, DC: American Psychiatric Association.

Americans With Disabilities Act of 1990, Pub. L. No. 101-336, 104 Stat. 328 (1990).

Brault, M. W. (July 2012). Americans with disabilities: 2010. Current Population Reports, P70-131. U.S. Census Bureau: Washington, DC.

Brommelhoff, J. A., Gatz, M., Johansson, B., McArdle, J. J., Fratiglioni, L., \& Pedersen, N. L. (2009). Depression as a risk factor or prodromal feature for dementia? Findings in a population-based sample of Swedish twins. Psychology and Aging, 24, 373-384. doi: $10.1037 / \mathrm{a} 0015713$

Centers for Disease Control and Prevention (CDC), National Center for Injury Prevention and Control. (2018). Web-based Injury Statistics Query and Reporting System (WISQARS) [online]. Available from URL: $\underline{\text { www.cdc.gov/ncipc/wisqars }}$

Chui, H., Gerstorf, D., Hoppmann, C. A., \& Luszcz, M. A. (2015). Trajectories of depressive symptoms in old age: Integrating age-, pathology-, and mortality-related changes. Psychology and Aging, 30, 940-951. doi: 10.1037/pag0000054

Conwell, Y., Duberstein, P. R., \& Caine, E. D. (2002). Risk factors for suicide in later life. 
Biological Psychiatry, 52, 193-204. doi: 10.1016/S0006-3223(02)01347-1

Conwell, Y., Duberstein, P. R., Hirsch, J. K., Conner, K. R., Eberly, S., \& Caine, E. D. (2010). Health status and suicide in the second half of life. International Journal of Geriatric Psychiatry, 25, 371-379. doi: 10.1002/gps.2348

Conwell, Y., Lyness, J. M., Duberstein, P., Cox, C., Seidlitz, L., DiGiorgio, A., \& Caine, E. D. (2000). Completed suicide among older patients in primary care practices: A controlled study. Journal of the American Geriatrics Society, 48, 23-29. doi: 10.1111/j.15325415.2000.tb03024.x

Conwell, Y., Van Orden, K., \& Caine, E. D. (2011). Suicide in older adults. Psychiatric Clinics of North America, 34, 451-468. doi: 10.1016/j.psc.2011.02.002

Cui, X., Lyness, J. M., Tang, W., Tu, X., \& Conwell, Y. (2008). Outcomes and predictors of late-life depression trajectories in older primary care patients. American Journal of Geriatric Psychiatry, 16, 406-415. doi: 10.1097/01.JGP.0000308881.22956.27

Drapeau, C. W., \& McIntosh, J. L. (for the American Association of Suicidology). (2015). U.S.A. suicide: 2013 official final data. Washington, DC: American Association of Suicidology, dated January 22, 2015. Retrieved from http://www.suicidology.org/Portals/14/docs/Resources/FactSheets/2013datapgs2alt.pdf

Fauth, E. B., Gerstorf, D., Ram, N., \& Malmberg, B. (2014). Comparing changes in late-life depressive symptoms across aging, disablement, and mortality processes. Developmental Psychology, 50, 1584-1593. doi: 10.1037/a0035475

Filiberti, A., Ripamonti, C., Totis, A., Ventafridda, V., De Conno, F., Contiero, P., \& Tamburini, M. (2001). Characteristics of terminal cancer patients who committed suicide during a home palliative care program. Journal of Pain and Symptom Management, 22, 544-553. 
doi: 10.1016/S0885-3924(01)00295-0

Haw, C., Harwood, D., \& Hawton, K. (2009). Dementia and suicidal behavior: a review of the literature. International Psychogeriatrics, 21, 440-453. doi:

$10.1017 / \mathrm{S} 1041610209009065$

He, W., \& Larsen, L. J. (2014). Older Americans With a Disability: 2008-2012 (U.S. Census Bureau American Community Survey Report ACS-29). U.S. Government Printing Office: Washington, DC.

Isometsä, E. T. (2001). Psychological autopsy studies - a review. European Psychiatry, 16, 379-385. doi: 10.1016/S0924-9338(01)00594-6

Jajodia, A. (2012). Dynamic structural equation models of change. In J.T. Newsom, R.N. Jones, \& S.M. Hofer (Eds.), Longitudinal data analysis (pp. 291-328). New York, NY: Routledge.

Kendler, K. S., Gatz, M., Gardner, C., \& Pedersen, N. A. (2006). Swedish national twin study of lifetime major depression. American Journal of Psychiatry, 163, 109-114. doi: 10.1176/appi.ajp.163.1.109

Kohout, F. J., Berkman, L. F., Evans, D. A., \& Cornoni-Huntley, J. (1993). Two shorter forms of the CES-D Depressive Symptoms Index. Journal of Aging and Health, 5, 179-193. doi: $10.1177 / 089826439300500202$

Lebowitz, B. D., Pearson, J. L., Schneider, L. S., Reynolds, C. F., Alexopoulos, G. S., Bruce, M. L., ..., Parmelee, P. (1997). Diagnosis and treatment of depression in late life. Journal of the American Medical Association, 278, 1186-1190.

Lenz, E. J., Schulz, R., Martire, L. M., Zdaniuk, B., Glass, T., Kop, W. J., ... \& Reynolds, C. F. (2005). The course of functional decline in older people with persistently elevated 
depressive symptoms: Longitudinal findings from the Cardiovascular Health Study. Journal of the American Geriatrics Society, 53, 569-575.

Leon, A. C., Friedman, R. A., Sweeney, J. A., Brown, R. P., \& Mann, J. J. (1990). Statistical issues in the identification of risk factors for suicidal behavior: The application of survival analysis. Psychiatry Research, 31, 99-108. doi: 10.1016/0165-1781(90)90112-I

Lichtenstein, P., De Faire, U., Floderus, B., Svartengren, M., Svedberg, P., \& Pedersen, N. L. (2002). The Swedish Twin Registry: a unique resource for clinical, epidemiological and genetic studies. Journal of Internal Medicine, 252, 184-205. doi: 10.1046/j.13652796.2002.01032.x

Lichtenstein, P., Sullivan, P. F., Cnattigius, S., Gatz, M., Johansson, S., Carlstrom, E., ..., Pedersen, N.L. (2006). The Swedish Twin Registry in the third millennium: An update. Twin Research and Human Genetics, 9, 875-882. doi: 10.1375/twin.9.6.875

Luoma, J. B., Martin, C. E., \& Pearson, J. L. (2002). Contact with mental health and primary care providers before suicide: A review of the evidence. American Journal of Psychiatry, 159, 909-916. doi: 10.1176/appi.ajp.159.6.909

Lutz, J., \& Fiske, A. (2017). Functional disability and suicidal behavior in middle-aged and older adults: A systematic critical review. Journal of Affective Disorders, 227, 260-271. doi: 10.1016/j.jad.2017.10.043

Park, S.- M. (2014). Health status and suicidal ideation in Korean elderly: the role of living arrangement. Journal of Mental Health, 23, 94-98. doi: 10.3109/09638237.2013.869569

Préville, M., Boyer, R., Hébert, R., Bravo, G., \& Seguin, M. (2005a). Correlates of suicide in the older adult population in Quebec. Suicide and Life-Threatening Behavior, 35, 91105. doi: $10.1521 /$ suli.35.1.91.59269 
Préville, M., Hébert, R., Boyer, R., Bravo, G., \& Seguin, M. (2005b). Physical health and mental disorder in elderly suicide: A case-control study. Aging \& Mental Health, 9, 576584. doi: 10.1080/13607860500192973

Rockett, I. R. H., Caine, E. D., Connery, H. S., \& Greenfield, S. F. (2018). Mortality in the United States from self-injury surpasses diabetes: a prevention imperative. Injury Prevention. Advance online publication. doi: 10.1136/injuryprev-2018-042889

Rockett, I. R. H., Kapusta, N. D., \& Bhandari, R. (2011). Suicide misclassification in an international context: Revisitation and update. Suicidology Online, 2, 48-61.

Rockett, I. R. H., Kapusta, N. D., \& Coben, J. H. (2014). Beyond suicide: Action needed to improve self-injury mortality accounting. JAMA Psychiatry, 71, 231-232. doi: 10.1001/jamapsychiatry.2013.3738

Rockett, I. R. H., Lilly, C. L., Jia, H., Larkin, G. L., Miller, T. R., Nelson, L. S., .. \& Caine, E. D. (2016). Self-injury mortality in the United States in the early 21 st century: a comparison with proximally ranked diseases. JAMA Psychiatry, 73, 1072-1081. doi: 10.1001/jamapsychiatry.2016.1870

Rockett, I. R. H., Smith, G. S., Caine, E.D., Kapusta, N. D., Hanzlick, R. L., Larkin, G. L., ... \& Fraser, D. W. (2014). Confronting death from drug self-intoxication (DDSI): prevention through a better definition. American Journal of Public Health, 104, e49-e55. doi: 10.2105/AJPH.2014.302244)

Rockett, I. R. H., Wang, S., Stack, S., De Leo, D., Frost, J. L., Ducatman, A. M., .. \& Kapusta, N. D. (2010). Race/ethnicity and potential suicide misclassification: Window on a minority suicide paradox? BMC Psychiatry, 10(35), 1-8. doi: 10.1001/jamapsychiatry.2013.3738 
Rubenowitz, E., Waern, M., Wilhelmson, K., \& Allebeck, P. (2001). Life events and psychosocial factors in elderly suicides - a case-control study. Psychological Medicine, 31, 1193-1202. doi: 10.1017\}S0033291701004457

Sbarra, D. A., \& Allen, J. J. B. (2009). Decomposing depression: On the prospective and reciprocal dynamics of mood and sleep disturbances. Journal of Abnormal Psychology, 118, 171-182. doi: 10.1037/a0014375

Serafini, G., Calcagno, P., Lester, D., Girardi, P., Amore, M., \& Pompili, M. (2016). Suicide risk in Alzheimer's disease: A systematic review. Current Alzheimer's Research, 13, 10831099. doi: $10.2174 / 15672050136661607201$

Singer, J. D., \& Willett, J. B. (2003). Applied longitudinal data analysis: Modeling change and event occurrence. New York: Oxford University Press.

Spector, W. D., \& Fleishman, J. A. (1998). Combining activities of daily living with instrumental activities of daily living to measure functional disability. Journal of Gerontology: Social Sciences, 53B, S46-S57. doi: 10.1093/geronb/53B.1.S46

Steffick, D. E. (2000). Documentation of affective functioning measures in the Health and Retirement Study. HRS/AHEAD Documentation Report DR-005. Retrieved from: http://hrsonline.isr.umich.edu/

Stone D. M., Holland, K. M., Bartholow, B., Logan, J. E., McIntosh, W. L., Trudeau, A., \& Rockett, I. R. H. (2017). Deciphering suicide and other manners of death associated with drug intoxication: a Centers for Disease Control and Prevention consultation meeting summary. American Journal of Public Health, 107, 1233-1239. doi: 10.2105/AJPH.2017.303863

Szanto, K., Lenze, E. J., Waern, M., Duberstein, P., Bruce, M. L., Epstein-Lubow, G., \& 
Conwell, Y. (2013). Research to reduce the suicide rate among older adults:

Methodology roadblocks and promising paradigms. Psychiatric Services, 64, 586-589. doi: 10.1176/appi.ps.003582012

Tsoh, J., Chiu, H. F. K., Duberstein, P. R., Chan, S. S. M., Chi, I., Yip, P. S. F., \& Conwell, Y. (2005). Attempted suicide in elderly Chinese persons: A multi-group, controlled study. American Journal of Geriatric Psychiatry, 13, 562-571. doi: 10.1097/00019442200507000-00004

U.K. Department of Health. (January 2014). Statistical update on suicide. Retrieved from https://www.gov.uk/government/uploads/system/uploads/attachment_data/file/278120/Su icide_update_Jan_2014_FINAL_revised.pdf

World Health Organization. (2002). Towards a common language for functioning, disability and health: ICF. Retrieved from http://www.who.int/classifications/icf/training/icfbeginnersguide.pdf

World Health Organization (WHO). (2014). Preventing suicide: A global imperative. Retrieved from http://www.who.int/mental_health/suicide-prevention/exe_summary_english.pdf Yang, Y. (2007). Is old age depression? Growth trajectories and cohort variations in late-life depression. Journal of Health and Social Behavior, 48, 16-32. doi: $10.1177 / 002214650704800102$ 
Table 1

Number of waves of data available for participants in the Health and Retirement Study (HRS)

Number Frequency of Percent of Cumulative Number Number of Number of drug of waves participants participants frequency of suicides undetermined intoxication

\begin{tabular}{|c|c|c|c|c|c|c|}
\hline & & & & & deaths & deaths \\
\hline 1 & 3429 & 9.74 & 3429 & 8 & 1 & 3 \\
\hline 2 & 3307 & 9.39 & 6736 & 6 & 0 & 2 \\
\hline 3 & 6996 & 19.86 & 13732 & 7 & 1 & 1 \\
\hline 4 & 2316 & 6.58 & 16048 & 2 & 0 & 1 \\
\hline 5 & 2232 & 6.34 & 18280 & 2 & 1 & 2 \\
\hline 6 & 3481 & 9.88 & 21761 & 2 & 1 & 0 \\
\hline 7 & 1846 & 5.24 & 23607 & 7 & 0 & 0 \\
\hline 8 & 1966 & 5.58 & 25573 & 2 & 0 & 0 \\
\hline 9 & 3301 & 9.37 & 28874 & & & \\
\hline 10 & 1916 & 5.44 & 30790 & & & \\
\hline 11 & 4432 & 12.58 & 35222 & & & \\
\hline
\end{tabular}


Table 2

Sample sizes and missing data on main variables in waves of Health and Retirement Study (HRS)

Depressive symptoms $\quad$ Functional limitations

\begin{tabular}{lccccc} 
Wave & $N$ & Missing, $n(\%)$ & M (SD) & Missing, $n(\%)$ & M (SD) \\
\hline $1994($ HRS) & 10823 & $854(7.9)$ & $2.95(1.42)$ & $180(1.7)$ & $2.94(3.09)$ \\
1995 (AHEAD) & 6964 & $877(12.6)$ & $3.12(1.46)$ & $133(1.9)$ & $4.23(4.33)$ \\
1996 & 10414 & $706(6.8)$ & $2.91(1.37)$ & $896(8.6)$ & $3.11(3.69)$ \\
1998 & 20635 & $2009(9.7)$ & $3.19(1.44)$ & $951(4.6)$ & $3.43(3.82)$ \\
2000 & 19019 & $2034(10.7)$ & $3.17(1.45)$ & $918(4.8)$ & $3.48(3.84)$ \\
2002 & 17681 & $1947(11.0)$ & $3.13(1.45)$ & $662(3.7)$ & $3.71(3.83)$ \\
2004 & 19108 & $1633(8.6)$ & $3.08(1.44)$ & $660(3.5)$ & $3.63(3.75)$ \\
2006 & 17930 & $1258(7.0)$ & $3.10(1.45)$ & $486(2.7)$ & $3.86(3.84)$ \\
2008 & 16857 & $1130(6.7)$ & $3.00(1.38)$ & $474(2.8)$ & $3.85(3.82)$ \\
2010 & 18289 & $1049(5.7)$ & $3.04(1.41)$ & $218(1.2)$ & $4.06(4.06)$ \\
2012 & 21034 & $1375(6.5)$ & $3.04(1.39)$ & $391(1.9)$ & $3.91(3.98)$ \\
\hline
\end{tabular}

Note. The number of individuals who participated in a given wave but are missing on totals of these variables in that wave is presented here. For the purposes of this study, the 1994 and 1995 waves were combined for analyses. 
Table 3

Results of univariate dual change score models (DCSM) in entire sample

\begin{tabular}{lcc} 
& ADL & CESD \\
\hline $\mathrm{N}$ & 34824 & 33466
\end{tabular}

Parameter estimates

\begin{tabular}{|c|c|c|}
\hline Mean InADL & $2.773 * * *$ & -- \\
\hline Mean SlADL & $-0.393 * * *$ & -- \\
\hline Mean InCESD & -- & $3.187 * * *$ \\
\hline Mean SICESD & -- & $0.407 * * *$ \\
\hline InADL on Sex & $-0.920 * * *$ & -- \\
\hline SIADL on Sex & $0.137 * * *$ & -- \\
\hline InCESD on Sex & -- & $-0.276 * * *$ \\
\hline SlCESD on Sex & -- & $-0.035 * * *$ \\
\hline InADL on Age & $0.064 * * *$ & -- \\
\hline SlADL on Age & $0.008 * * *$ & -- \\
\hline InCESD on Age & -- & $0.003 * * *$ \\
\hline SlCESD on Age & -- & $0.005 * * *$ \\
\hline$\triangle \mathrm{ADL} 2$ on $\mathrm{ADL} 1$ & $0.152 * * *$ & -- \\
\hline$\triangle \mathrm{CESD} 2$ on $\mathrm{CESD} 1$ & -- & $-0.137 * * *$ \\
\hline InADL with SIADL & $-1.530 * * *$ & -- \\
\hline InCESD with SICESD & -- & $0.103^{* * *}$ \\
\hline
\end{tabular}




\section{Model fit indices}

$\begin{array}{lcc}\text { AIC } & 913075.290 & 583796.830 \\ \text { BIC } & 913168.328 & 583889.431 \\ \text { Sample-size adjusted BIC } & 913133.370 & 583854.473 \\ \text { RMSEA (90\% CI) } & .039(.038-.040) & .017(.016-.018) \\ \text { CFI } & .949 & .980 \\ \text { TLI } & .956 & .982 \\ \text { SRMR } & .064 & .037\end{array}$

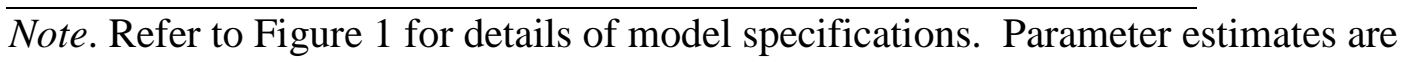
unstandardized. InADL = intercept growth parameter for ADL; InCESD = intercept growth parameter for CESD; SIADL = slope growth parameter for ADL; SICESD = slope growth parameter for CESD; ADL1 = latent level of functional disability at preceding time point; $\triangle \mathrm{ADL} 2=$ latent change from ADL1 to next time point; CESD1 = latent level of depressive symptoms at preceding time point; $\triangle \mathrm{CESD} 2=$ latent change from CESD1 to next time point. $\mathrm{AIC}=$ Akaike information criterion; $\mathrm{BIC}=$ Bayesian information criterion; $\mathrm{RMSEA}=$ root mean square error of approximation; $\mathrm{CI}=$ confidence interval; $\mathrm{CFI}=$ comparative fit index; $\mathrm{TLI}=$ Tucker-Lewis index; SRMR = standardized root mean square residual.

$* p<.05$

$* * p<.01$

$* * * p<.001$ 
Table 4

Results of bivariate dual change score models (DCSM) in entire sample

\begin{tabular}{ccc}
$\begin{array}{c}\text { No paths (neither } \\
\text { a nor b) }\end{array}$ & ADL to CESD & CESD to ADL \\
(a paths) & (b paths) \\
\hline 34901 & 34901 & 34901
\end{tabular}

\begin{tabular}{llll}
\hline $\mathrm{N}$ & 34901 & 34901 & 34901
\end{tabular}

\section{Parameter estimates}

\begin{tabular}{|c|c|c|c|}
\hline Mean InADL & $2.777 * * *$ & $2.777 * * *$ & $2.842 * * *$ \\
\hline Mean SIADL & $-0.384 * * *$ & $-0.386 * * *$ & $5.667 * * *$ \\
\hline Mean InCESD & $3.152 * * *$ & $3.157 * * *$ & $3.102 * * *$ \\
\hline Mean SICESD & $0.209 * * *$ & $0.293 * * *$ & -0.060 \\
\hline InADL on Sex & $-0.919 * * *$ & $-0.920 * * *$ & $-0.936 * * *$ \\
\hline SIADL on Sex & $0.135^{* * *}$ & $0.136^{* * *}$ & $-0.252 * * *$ \\
\hline InCESD on Sex & $-0.279 * * *$ & $-0.279 * * *$ & $-0.277 * * *$ \\
\hline SlCESD on Sex & $-0.018 * *$ & $-0.021 * * *$ & 0.007 \\
\hline InADL on Age & $0.063 * * *$ & $0.063 * * *$ & $0.060 * * *$ \\
\hline SlADL on Age & $0.009 * * *$ & $0.009 * * *$ & $0.028 * * *$ \\
\hline InCESD on Age & $0.008 * * *$ & $0.008 * * *$ & $0.013 * * *$ \\
\hline SlCESD on Age & $0.006^{* * *}$ & $0.006^{* * *}$ & $0.003 * * *$ \\
\hline$\triangle \mathrm{ADL} 2$ on $\mathrm{ADL} 1$ & $0.148 * * *$ & $0.149 * * *$ & $0.363 * * *$ \\
\hline$\triangle \mathrm{CESD} 2$ on CESD1 & $-0.075 * * *$ & $-0.109 * * *$ & 0.018 \\
\hline$\triangle \mathrm{CESD} 2$ on ADL1 (a) & -- & $0.007 *$ & -- \\
\hline$\triangle \mathrm{ADL} 2$ on CESD1 (b) & -- & -- & $-2.162 * * *$ \\
\hline
\end{tabular}




$\begin{array}{lccc}\text { InADL with SIADL } & -1.501 * * * & -1.507 * * * & 1.092 * * * \\ \text { InCESD with SICESD } & 0.052 * * & 0.072 * * & -0.031 \\ \text { InADL with InCESD } & 2.330 * * * & 2.328 * * * & 2.284 * * * \\ \text { SIADL with SICESD } & 0.001 & -0.001 & -0.003 \\ \text { InADL with SICESD } & 0.140 * * * & 0.150 * * * & -0.066 \\ \text { SIADL with InCESD } & -0.361 * * * & -0.362 * * * & 1.494 * * *\end{array}$

\section{Model fit indices}

$\begin{array}{lccc}\text { AIC } & 1483188.180 & 1483181.414 & 1482010.505 \\ \text { BIC } & 1483408.147 & 1483409.841 & 1482238.933 \\ \text { Sample-size adjusted BIC } & 1483325.519 & 1483324.035 & 1482153.127 \\ \text { RMSEA }(90 \% \mathrm{CI}) & .028(.027-.028) & .028(.027-.028) & .027(.026-.027) \\ \text { CFI } & .951 & .951 & .955 \\ \text { TLI } & .954 & .953 & .958 \\ \text { SRMR } & .045 & .046 & .039 \\ \text { Loglikelihood difference test }^{\mathrm{a}} & & 10.4612^{* *} & 190.0585^{* * *}\end{array}$

Note. Refer to Figure 2 for details of model specifications. Parameter estimates are unstandardized. Bidirectional bivariate model (i.e. including both a and b paths; see Appendix G) did not converge. InADL = intercept growth parameter for ADL; InCESD = intercept growth parameter for CESD; SIADL = slope growth parameter for ADL; SICESD = slope growth parameter for CESD; ADL1 = latent level of functional disability at preceding time point; $\triangle \mathrm{ADL} 2=$ latent change from ADL1 to next time point; CESD1 = latent level of depressive symptoms at preceding time point; $\triangle \mathrm{CESD} 2=$ latent change from CESD1 to next time point. 
$\mathrm{AIC}=$ Akaike information criterion $; \mathrm{BIC}=$ Bayesian information criterion; $\mathrm{RMSEA}=$ root mean square error of approximation; $\mathrm{CI}=$ confidence interval; $\mathrm{CFI}=$ comparative fit index; $\mathrm{TLI}=$ Tucker-Lewis index; SRMR = standardized root mean square residual.

${ }^{a}$ Compared to the no-paths model. The loglikelihood difference test utilizes the following formulas to test significance of differences in loglikelihood for nested models: $\mathrm{Cd}=(\mathrm{p} 0 * \mathrm{c} 0-$ $\left.\mathrm{p} 1{ }^{*} \mathrm{c} 1\right) /(\mathrm{p} 0-\mathrm{p} 1) ;$ Chi-square difference test $=-2(\mathrm{~L} 0-\mathrm{L} 1) / \mathrm{cd} ; \mathrm{Df}=\mathrm{p} 1-\mathrm{p} 0$. The degrees of freedom for the loglikelihood difference test for both of these models were 1.

$* p<.05$

$* * p<.01$

$* * * p<.001$ 
Table 5

Comparison of fits of latent class models based on ADL-to-CESD bivariate dual change score model

\begin{tabular}{lccc} 
& 2 class & 3 class & 4 class $^{\mathrm{a}}$ \\
\hline BIC & 1473896.537 & 1462520.713 & 1455574.055 \\
Sample-size adjusted BIC & 1473785.307 & 1462384.060 & 1455411.977 \\
LMR-LRT & $9283.657 * * *$ & $10587.625 * * *$ & $6947.320 * * *$ \\
Entropy & .721 & .738 & .654 \\
N (\%) Class 1 & $2505(7.2 \%)$ & $5486(15.7 \%)$ & $8880(25.4 \%)$ \\
N (\%) Class 2 & $32396(92.8 \%)$ & $25783(73.9 \%)$ & $4438(12.7 \%)$ \\
N (\%) Class 3 & -- & $3632(10.4 \%)$ & $19650(56.3 \%)$ \\
N (\%) Class 4 & - & - & $1933(5.5 \%)$
\end{tabular}

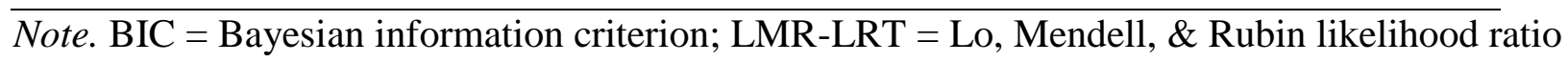
test; BLRT = bootstrap likelihood ratio test.

${ }^{\text {a }}$ The best loglikelihood value for the 4-class model did not replicate, and therefore the results may represent a local solution, and not a trustworthy solution. 
Table 6

Results of three-class latent class analysis based on ADL-to-CESD bivariate dual change score model

\begin{tabular}{|c|c|c|c|}
\hline & Class 1 & Class 2 & Class 3 \\
\hline$N$ & 5486 & 25783 & 3632 \\
\hline Mean InADL & $8.725 * * *$ & $1.659 * * *$ & $1.704 * * *$ \\
\hline Mean SIADL & $-1.288 * * *$ & $-0.094 *$ & $-0.186 * * *$ \\
\hline Mean InCESD & $5.035 * * *$ & $2.768 * * *$ & $3.315^{* * *}$ \\
\hline Mean SICESD & $0.912 * * *$ & $1.292 * * *$ & $0.532 * * *$ \\
\hline InADL on $\mathrm{Sex}^{\mathrm{a}}$ & $-0.536 * * *$ & $-0.536 * * *$ & $-0.536 * * *$ \\
\hline S1ADL on Sex ${ }^{a}$ & $0.045 * *$ & $0.045 * *$ & $0.045^{* *}$ \\
\hline InCESD on $\mathrm{Sex}^{\mathrm{a}}$ & $-0.166 * * *$ & $-0.166 * * *$ & $-0.166 * * *$ \\
\hline S1CESD on $\operatorname{Sex}^{\mathrm{a}}$ & $-0.058 * * *$ & $-0.058 * * *$ & $-0.058 * * *$ \\
\hline InADL on $\mathrm{Age}^{\mathrm{a}}$ & $0.046^{* * *}$ & $0.046 * * *$ & $0.046^{* * *}$ \\
\hline SlADL on Age ${ }^{a}$ & $0.008 * * *$ & $0.008 * * *$ & $0.008 * * *$ \\
\hline InCESD on $\mathrm{Age}^{\mathrm{a}}$ & -0.002 & -0.002 & -0.002 \\
\hline SlCESD on Age $^{a}$ & $0.006 * * *$ & $0.006 * * *$ & $0.006^{* * *}$ \\
\hline$\triangle \mathrm{ADL} 2$ on $\mathrm{ADL} 1$ & $0.146 * * *$ & $0.086 * * *$ & $0.351 * * *$ \\
\hline$\triangle \mathrm{CESD} 2$ on $\mathrm{CESD} 1$ & $-0.173 * * *$ & $-0.525 * * *$ & $-0.122 * * *$ \\
\hline$\triangle \mathrm{CESD} 2$ on ADL1 & $-0.020 * *$ & $0.052 * * *$ & $0.021 * * *$ \\
\hline InADL with SIADL ${ }^{a}$ & $-0.318 * * *$ & $-0.318 * * *$ & $-0.318 * * *$ \\
\hline InCESD with SICESD ${ }^{a}$ & $0.121 * * *$ & $0.121 * * *$ & $0.121 * * *$ \\
\hline
\end{tabular}




\begin{tabular}{|c|c|c|c|}
\hline InADL with InCESD ${ }^{a}$ & $0.265^{* * *}$ & $0.265 * * *$ & $0.265 * * *$ \\
\hline SIADL with SICESD ${ }^{\mathrm{a}}$ & -0.003 & -0.003 & -0.003 \\
\hline InADL with SICESD ${ }^{a}$ & $0.139 * * *$ & $0.139 * * *$ & $0.139 * * *$ \\
\hline SlADL with InCESD ${ }^{a}$ & $-0.041 * * *$ & $-0.041 * * *$ & $-0.041 * * *$ \\
\hline
\end{tabular}

$\overline{\text { Note. } \text { Refer to Figure } 2 \text { for details of overall model specifications and class specifications. }}$

Parameter estimates are unstandardized. InADL = intercept growth parameter for ADL; InCESD $=$ intercept growth parameter for CESD; SIADL = slope growth parameter for ADL; SICESD = slope growth parameter for CESD; ADL1 = latent level of functional disability at preceding time point; $\triangle \mathrm{ADL} 2=$ latent change from ADL1 to next time point; CESD1 = latent level of depressive symptoms at preceding time point; $\triangle \mathrm{CESD} 2=$ latent change from CESD1 to next time point.

a These parameters were constrained to be the same across classes.

$* p<.05$

$* * p<.01$

$* * * p<.001$ 
Table 7

Survival analysis results in Health and Retirement Study (HRS)

Suicide

Suicide \& undetermined

Suicide, undetermined, \& drug

intoxication

$\underline{\text { Rates by age band }}$

Age 50-59

Age 60-69

Age 70-79

Age 80 and older

$\underline{\text { Model } 1}$

$\operatorname{Sex}(1=$ male $)$

Age band

Age 60-69

Age 70-79

Age 80 and older

Rate/100,000 [95\% C.I.]

9.13 [4.35-19.16]

6.33 [3.02-13.28]

11.06 [5.76-21.26]

13.98 [6.66-29.32]

Rate/100,000 [95\% C.I.]

9.13 [4.35-19.16]

7.23 [3.62-14.46]

12.29 [6.61-22.85]

15.98 [7.99-31.95]
Rate/100,000 [95\% C.I.]

13.05 [7.02-24.25]

8.14 [4.23-15.64]

$12.29[6.61-22.85]$

23.97 [13.62-42.20] 


\begin{tabular}{|c|c|c|c|c|c|c|}
\hline Class 1 & 4.06 [1.57-10.49] & $4.11(1)^{*}$ & $4.31[1.76-10.52]$ & $4.91(1)^{*}$ & $4.00[1.80-8.87]$ & $5.78(1)^{*}$ \\
\hline Class 3 & $2.38[0.74-7.61]$ & $1.85(1)$ & $2.26[0.73-6.95]$ & $1.91(1)$ & $1.69[0.58-4.91]$ & $1.06(1)$ \\
\hline QIC & 198.60 & & 221.29 & & 260.56 & \\
\hline$\underline{\text { Model } 2}$ & IRR [95\% C.I.] & $\chi^{2}(d f)$ & $\operatorname{IRR}[95 \%$ C.I.] & $\chi^{2}(d f)$ & IRR [95\% C.I.] & $\chi^{2}(d f)$ \\
\hline Sex $(1=$ male $)$ & $3.57[1.62-7.86]$ & $8.94(1)^{* *}$ & $3.55[1.68-7.51]$ & $9.87(1)^{* *}$ & $3.80[1.93-7.50]$ & $13.12(1) * * *$ \\
\hline Age band & & $2.68(3)$ & & $3.09(3)$ & & $5.78(3)$ \\
\hline Age $60-69$ & $0.71[0.25-2.02]$ & & $0.82[0.29-2.24]$ & & $0.63[0.26-1.56]$ & \\
\hline Age $70-79$ & $1.31[0.49-3.54]$ & & $1.47[0.56-3.89]$ & & $1.04[0.43-2.50]$ & \\
\hline Age 80 and older & $1.90[0.65-5.54]$ & & $2.22[0.79-6.23]$ & & $2.42[1.03-5.69]$ & \\
\hline Class 1 & 4.93 [1.89-12.87] & $4.73(1)^{*}$ & $5.29[2.15-13.03]$ & $5.62(1)^{*}$ & $4.98[2.22-11.14]$ & $6.78(1)^{* *}$ \\
\hline Class 3 & $3.68[1.12-12.06]$ & $3.21(1)^{\dagger}$ & 3.56 [1.13-11.19] & $3.52(1)^{\dagger}$ & $2.71[0.92-8.03]$ & $2.94(1)^{\dagger}$ \\
\hline Cognitive impairment & $0.15[0.02-1.19]$ & $7.48(1)^{* *}$ & $0.14[0.02-1.05]$ & $9.23(1)^{* *}$ & $0.11[0.01-0.85]$ & $13.17(1)^{* * *}$ \\
\hline
\end{tabular}




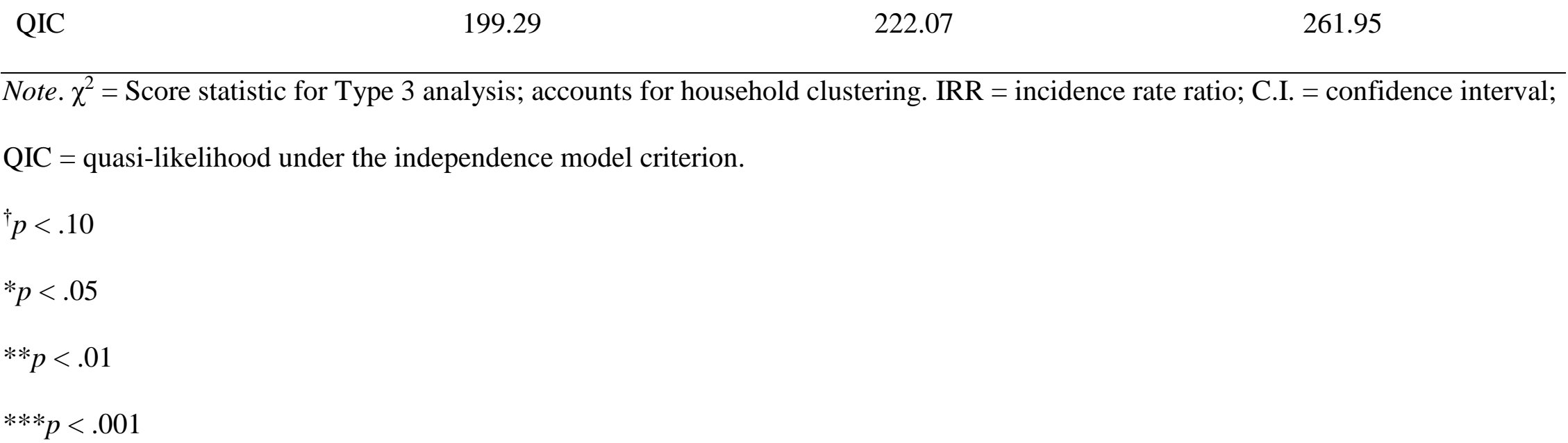


Table 8

Survival analysis in HRS, excluding participants with cognitive impairment.

Suicide $\quad$ Suicide \& undetermined
Suicide, undetermined, \& drug

intoxication

\begin{tabular}{|c|c|c|c|c|c|c|}
\hline Rates by age band & \multicolumn{2}{|c|}{ Rate/100,000 [95\% C.I.] } & \multicolumn{2}{|c|}{ Rate/100,000 [95\% C.I.] } & \multicolumn{2}{|c|}{ Rate/100,000 [95\% C.I.] } \\
\hline Age 50-59 & \multicolumn{2}{|c|}{$9.33[4.45-19.56]$} & \multicolumn{2}{|c|}{$9.33[4.45-19.56]$} & \multicolumn{2}{|c|}{$13.32[7.17-24.76]$} \\
\hline Age 60-69 & \multicolumn{2}{|c|}{$5.68[2.55-12.65]$} & \multicolumn{2}{|c|}{$6.63[3.16-13.91]$} & \multicolumn{2}{|c|}{$7.58[3.79-15.15]$} \\
\hline Age $70-79$ & \multicolumn{2}{|c|}{$12.46[6.48-23.95]$} & \multicolumn{2}{|c|}{$13.85[7.45-25.73]$} & \multicolumn{2}{|c|}{$13.85[7.45-25.73]$} \\
\hline Age 80 and older & \multicolumn{2}{|c|}{$19.09[9.10-40.04]$} & \multicolumn{2}{|c|}{21.82 [10.91-43.63] } & \multicolumn{2}{|c|}{32.73 [18.59-57.63] } \\
\hline$\underline{\text { Model }}$ & $\operatorname{IRR}[95 \%$ C.I.] & $\chi^{2}(d f)$ & $\operatorname{IRR}[95 \%$ C.I.] & $\chi^{2}(d f)$ & $\operatorname{IRR}[95 \%$ C.I.] & $\chi^{2}(d f)$ \\
\hline $\operatorname{Sex}(1=$ male $)$ & 3.95 [1.74-8.99] & $9.59(1)^{* *}$ & $3.88[1.78-8.46]$ & $10.53(1)^{* *}$ & $4.10[2.03-8.26]$ & $13.77(1)^{* * *}$ \\
\hline Age band & & $3.99(3)$ & & $4.27(3)$ & & $7.23(3)^{\dagger}$ \\
\hline Age 60-69 & $0.61[0.20-1.81]$ & & $0.71[0.25-2.03]$ & & $0.57[0.22-1.43]$ & \\
\hline Age 70-79 & $1.33[0.49-3.58]$ & & $1.49[0.57-3.93]$ & & $1.05[0.43-2.52]$ & \\
\hline Age 80 and older & $2.01[0.70-5.80]$ & & $2.33[0.84-6.49]$ & & $2.52[1.08-5.88]$ & \\
\hline
\end{tabular}




$\begin{array}{lllllll}\text { Class } 1 & 4.47[1.64-12.21] & 3.79(1)^{\dagger} & 4.88[1.91-12.46] & 4.67(1)^{*} & 4.68[2.04-10.73] & 5.84(1)^{*} \\ \text { Class } 3 & 4.02[1.24-13.00] & 3.57(1)^{\dagger} & 3.85[1.24-11.98] & 3.88(1)^{*} & 2.90[0.99-8.51] & 3.26(1)^{\dagger}\end{array}$

$\begin{array}{llll}\text { QIC } & 187.69 & 210.64 & 251.22\end{array}$

Note. $\chi^{2}=$ Score statistic for Type 3 analysis; accounts for household clustering. ADLs = activities of daily living; IRR = incidence rate ratio; C.I. = confidence interval; $\mathrm{QIC}=$ quasi-likelihood under the independence model criterion.

$$
\begin{aligned}
& { }^{\dagger} p<.10 \\
& * p<.05 \\
& * * p<.01 \\
& * * * p<.001
\end{aligned}
$$


Table 9

Survival analysis results in Swedish Twin Registry (STR)

Suicide

Suicide \& undetermined
Suicide, undetermined, \& drug

intoxication

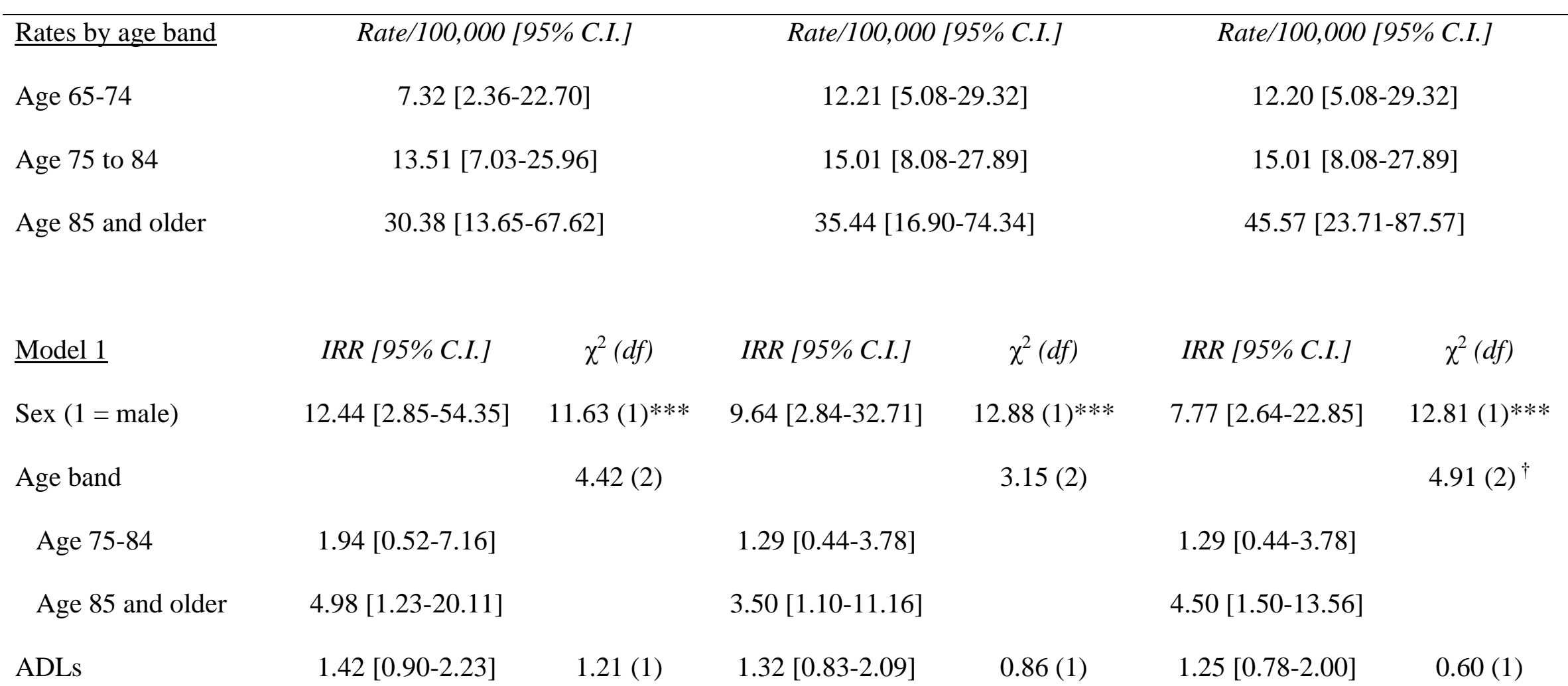




\begin{tabular}{|c|c|c|c|c|c|c|}
\hline QIC & \multicolumn{2}{|l|}{81.68} & \multicolumn{2}{|c|}{101.67} & \multicolumn{2}{|c|}{132.61} \\
\hline$\underline{\text { Model } 2}$ & $\operatorname{IRR}[95 \%$ C.I.] & $\chi^{2}(d f)$ & IRR [95\% C.I.] & $\chi^{2}(d f)$ & $\operatorname{IRR}[95 \%$ C.I.] & $\chi^{2}(d f)$ \\
\hline $\operatorname{Sex}(1=$ male $)$ & $15.36[3.45-68.29]$ & $11.97(1)^{* * *}$ & $11.88[3.44-41.05]$ & $13.20(1)^{* * *}$ & 9.89 [3.30-29.61] & $13.45(1)^{* * *}$ \\
\hline Age $75-84$ & $1.93[0.52-7.13]$ & & $1.29[0.44-3.77]$ & & $1.29[0.44-3.77]$ & \\
\hline Age 85 and older & $4.91[1.21-19.84]$ & & $3.46[1.08-11.01]$ & & 4.44 [1.47-13.39] & \\
\hline ADLs & $1.24[0.76-2.01]$ & $0.60(1)$ & $1.15[0.70-1.88]$ & $0.28(1)$ & $1.06[0.64-1.76]$ & $0.06(1)$ \\
\hline QIC & 110.67 & & 113. & & 139. & \\
\hline
\end{tabular}

Note. $\chi^{2}=$ Score statistic for Type 3 analysis; accounts for household clustering. ADLs = activities of daily living; IRR = incidence rate ratio; C.I. = confidence interval; $\mathrm{QIC}=$ quasi-likelihood under the independence model criterion.

$$
\begin{aligned}
& { }^{\dagger} p<.10 \\
& * p<.05 \\
& * * p<.01 \\
& * * * p<.001
\end{aligned}
$$




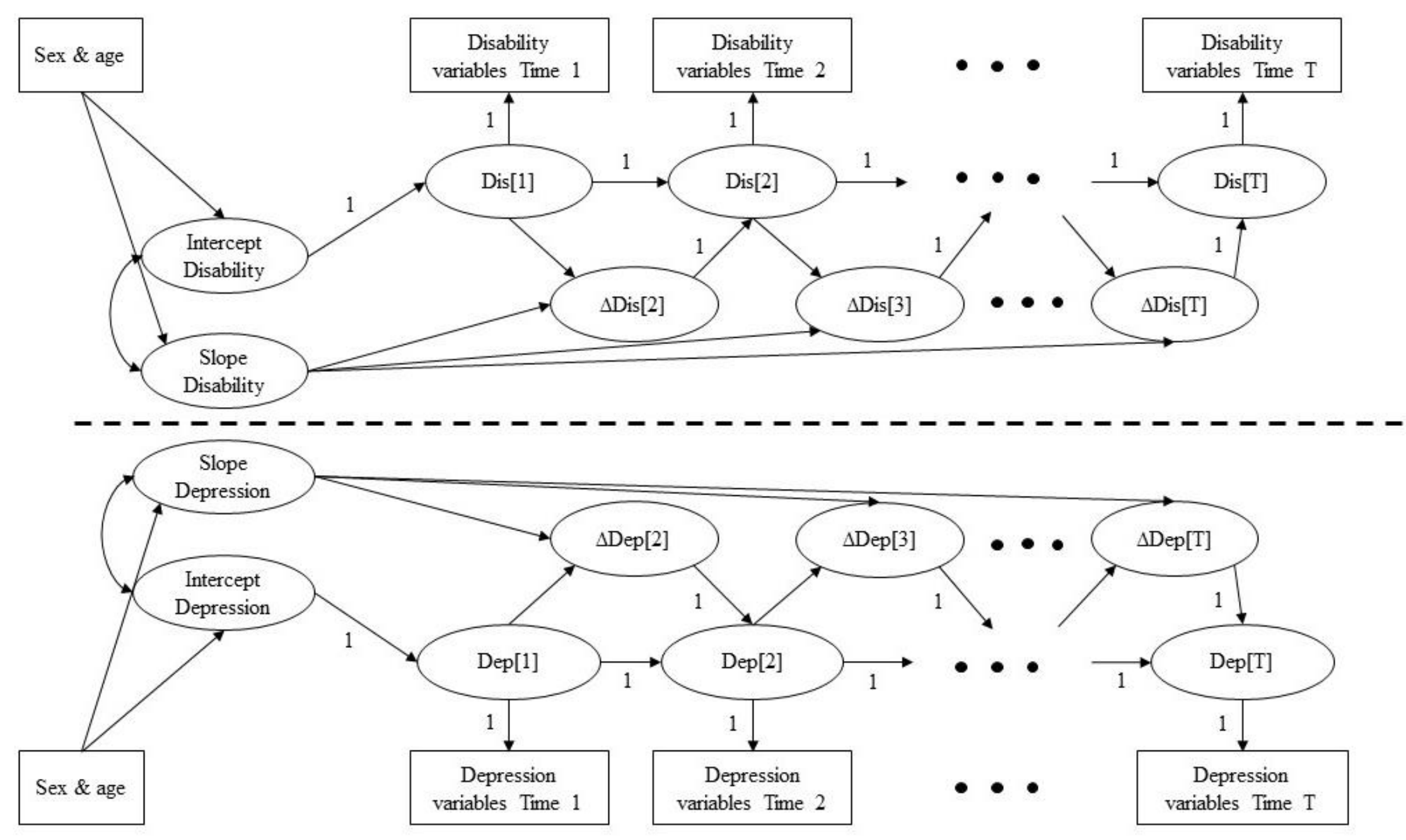


Figure 1. Diagram adapted from Jajodia (2012). Separate univariate dual change score models of longitudinal change/growth in disability and depression. For simplicity, error/residual terms are omitted from this diagram. In addition, measured variables labeled here as "Disability or depression variables Time T" represent a number of measured variables underlying the latent disability or depression scores at each time point. $\operatorname{Dis}[\mathrm{T}]=$ latent disability score at time $\mathrm{T} ; \mathrm{Dep}[\mathrm{T}]=$ latent depression score at time $\mathrm{T} ; \Delta \mathrm{Dis}[\mathrm{T}]=$ change in latent disability score from time $\mathrm{T}-1$ to time $\mathrm{T} ; \Delta \mathrm{Dep}[\mathrm{T}]=$ change in latent depression score from time $\mathrm{T}-1$ to time $\mathrm{T}$. 


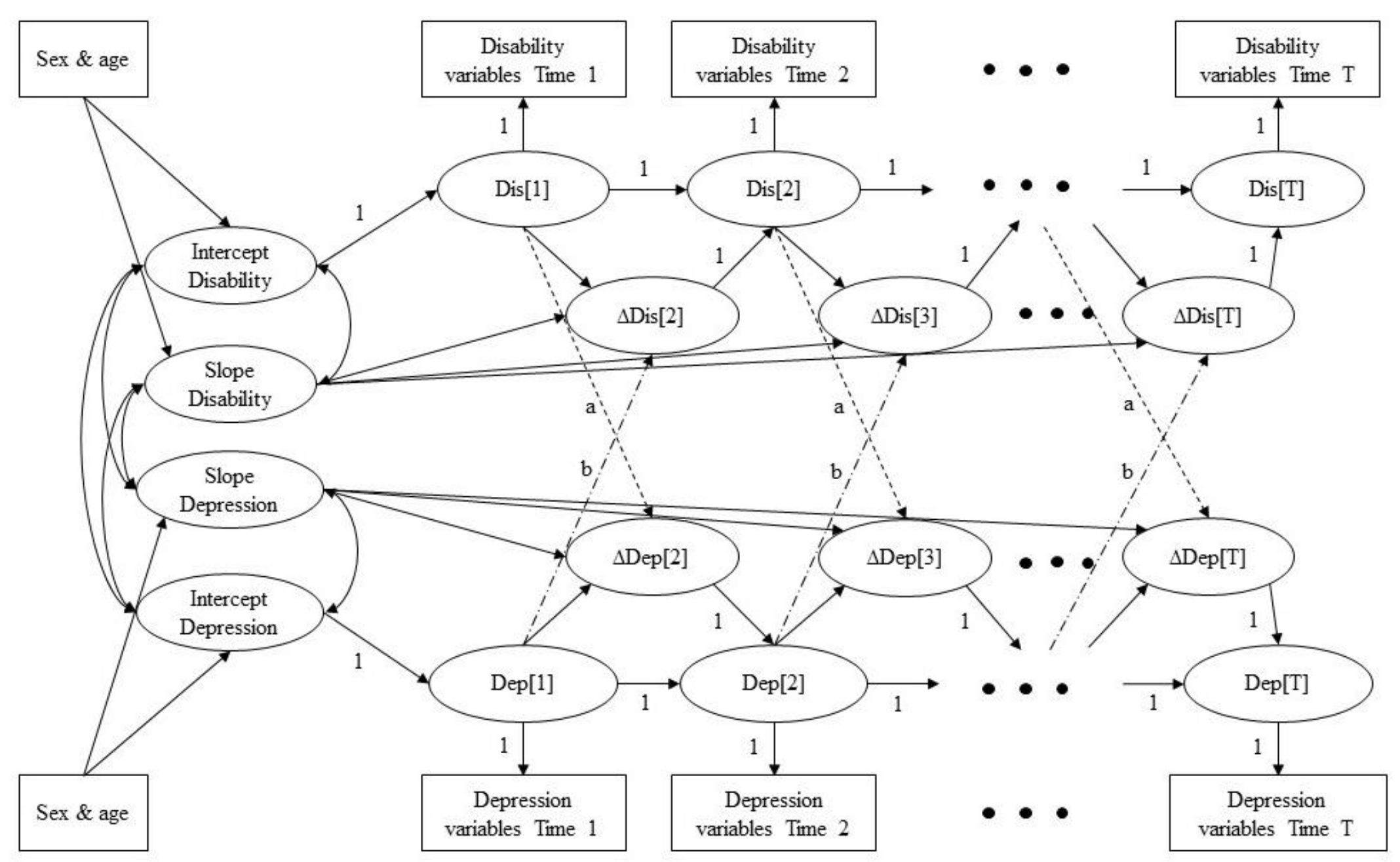


Figure 2. Diagram adapted from Jajodia (2012). Bivariate model incorporating the dual change score models for disability and depression from Figure 1. Paths labeled "a" represent paths where level of disability at time T predict change in depression from time $\mathrm{T}$ to time $\mathrm{T}+1$. Paths labeled " $\mathrm{b}$ " represent paths where level of depression at time $\mathrm{T}$ predict change in disability from time $\mathrm{T}$ to time $\mathrm{T}+1$. 
(a)

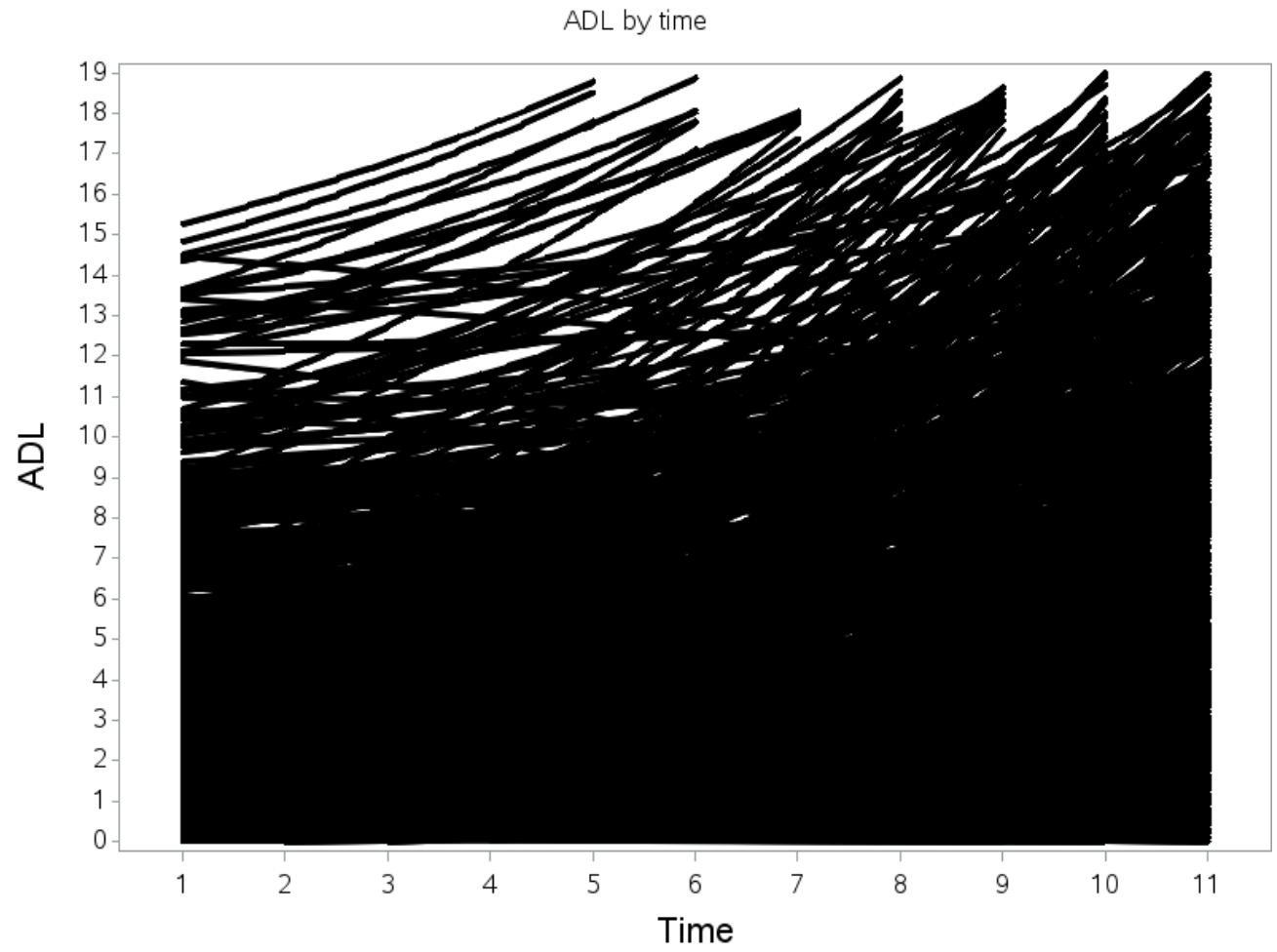

(b)

CESD by time

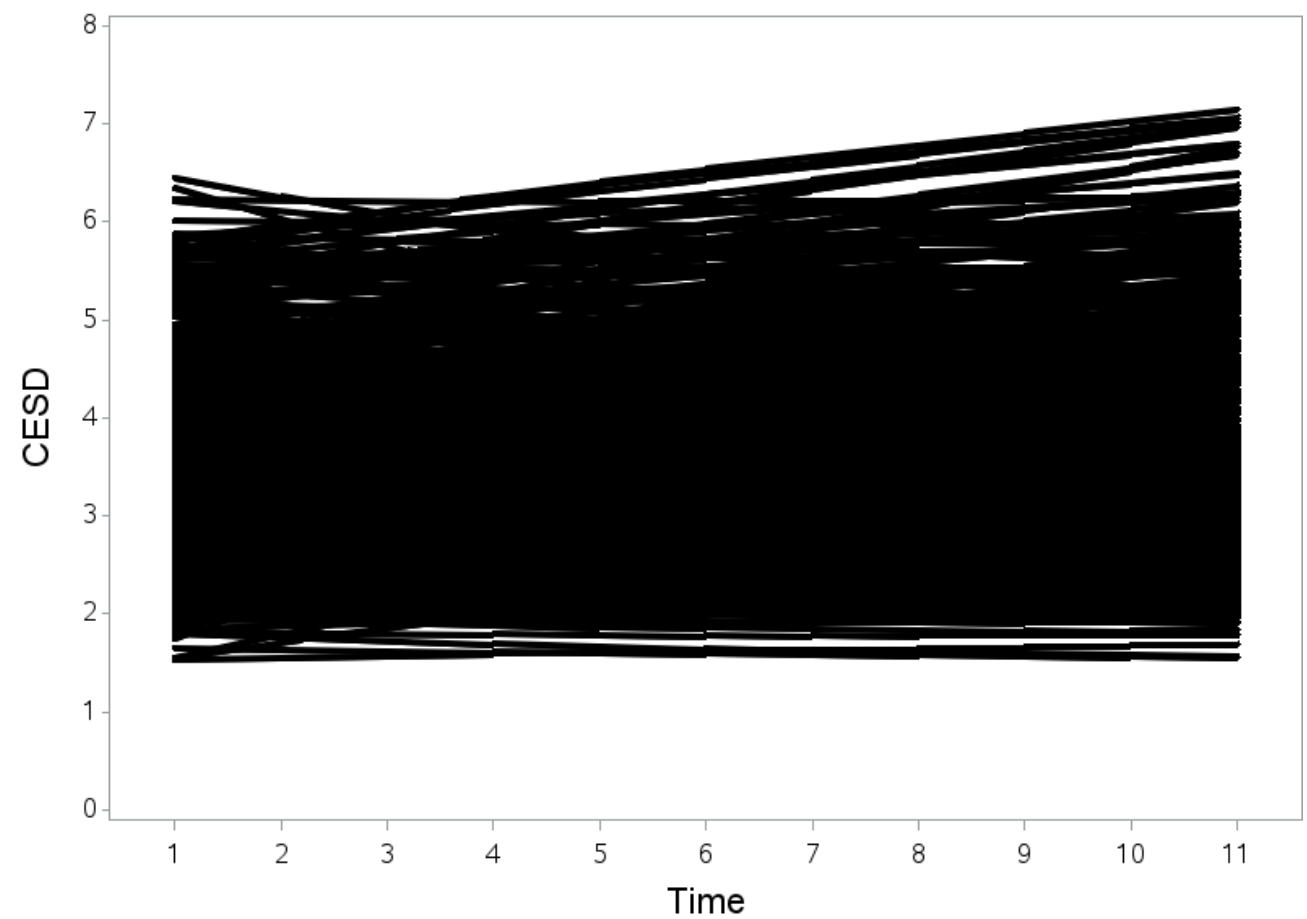


(c)

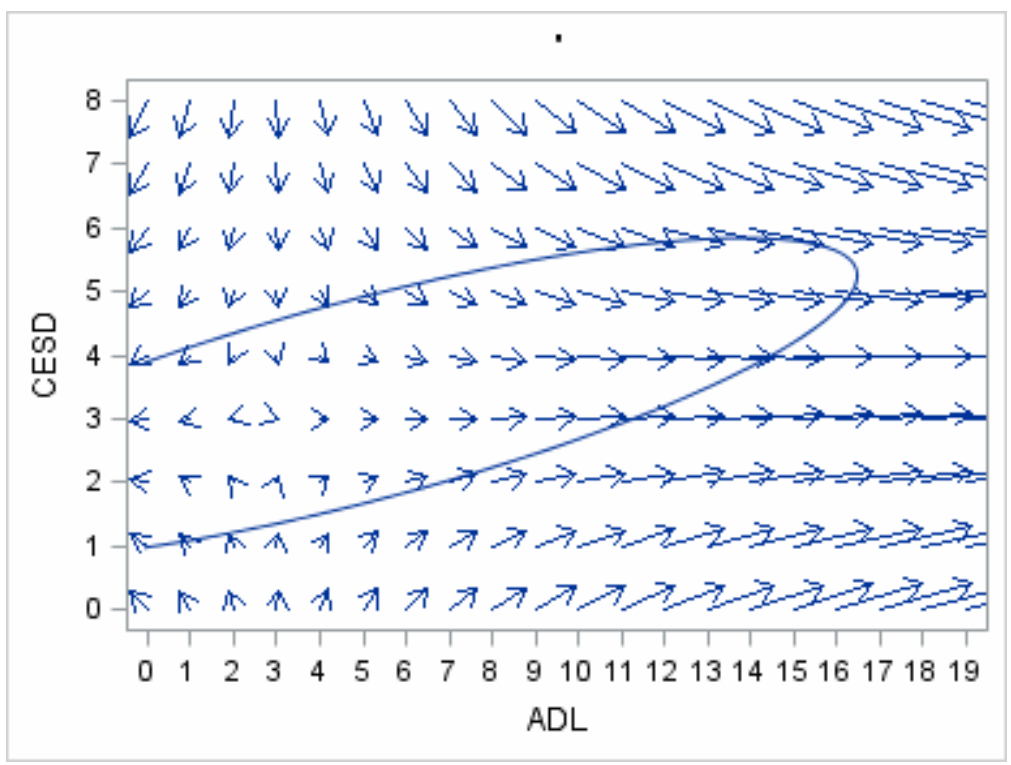

Figure 3. Plotting of change in ADLs and CESD over time and interaction between change in ADLs and CESD based on estimates from ADL-to-CESD bivariate dual change score model. (a) Plot of estimated ADL scores over time; (b) Plot of estimated CESD scores over time; (c) Vector plot of direction of change in ADL and CESD scores at different levels of each variable. 
(a)

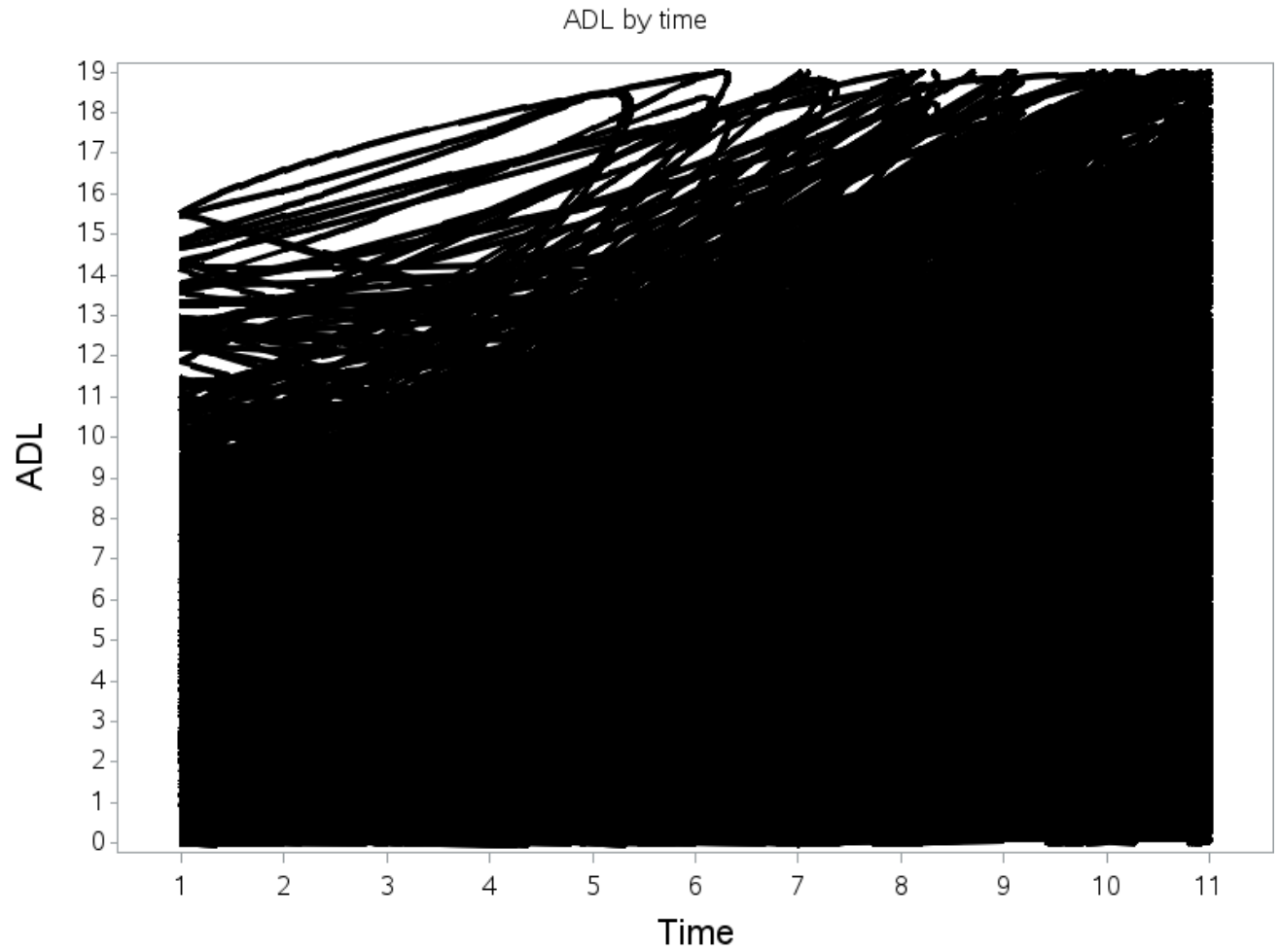

(b)

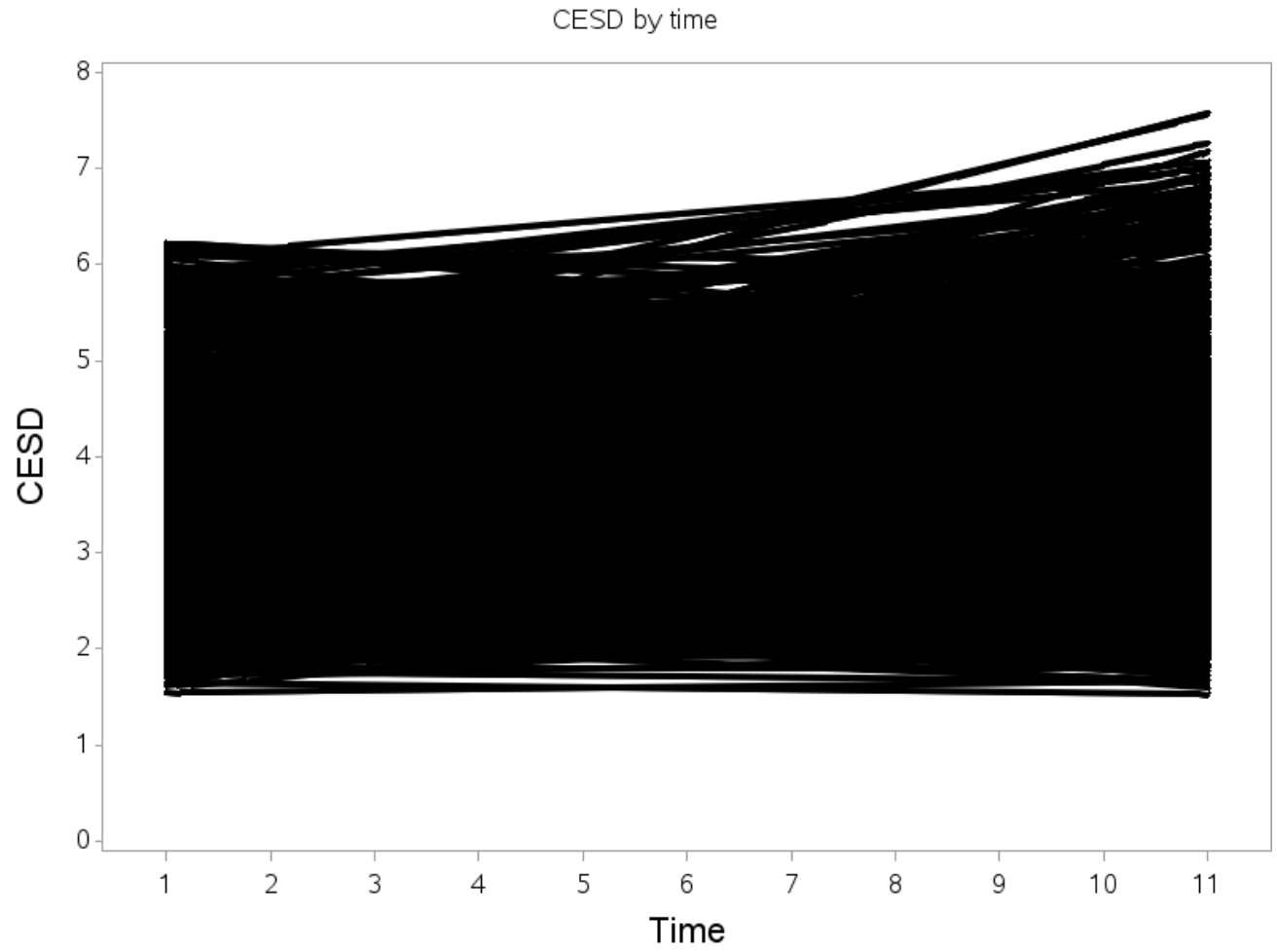


(c)

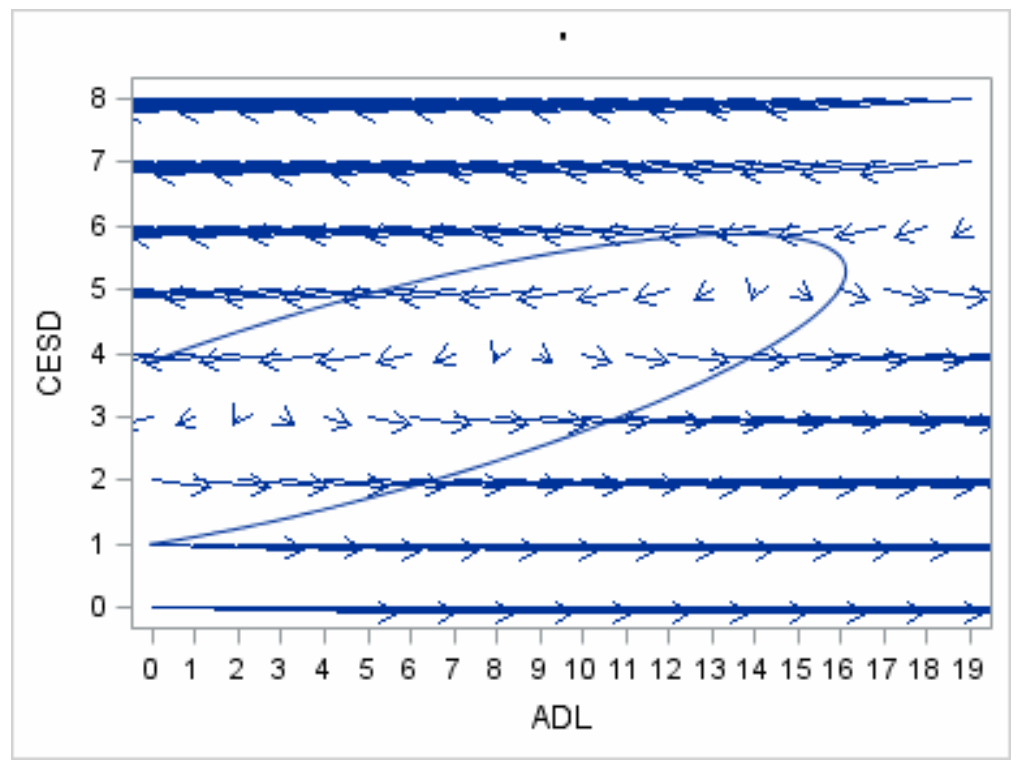

Figure 4. Plotting of change in ADLs and CESD over time and interaction between change in ADLs and CESD based on estimates from CESD-to-ADL bivariate dual change score model. (a) Plot of estimated ADL scores over time; (b) Plot of estimated CESD scores over time; (c) Vector plot of direction of change in ADL and CESD scores at different levels of each variable. 
(a)

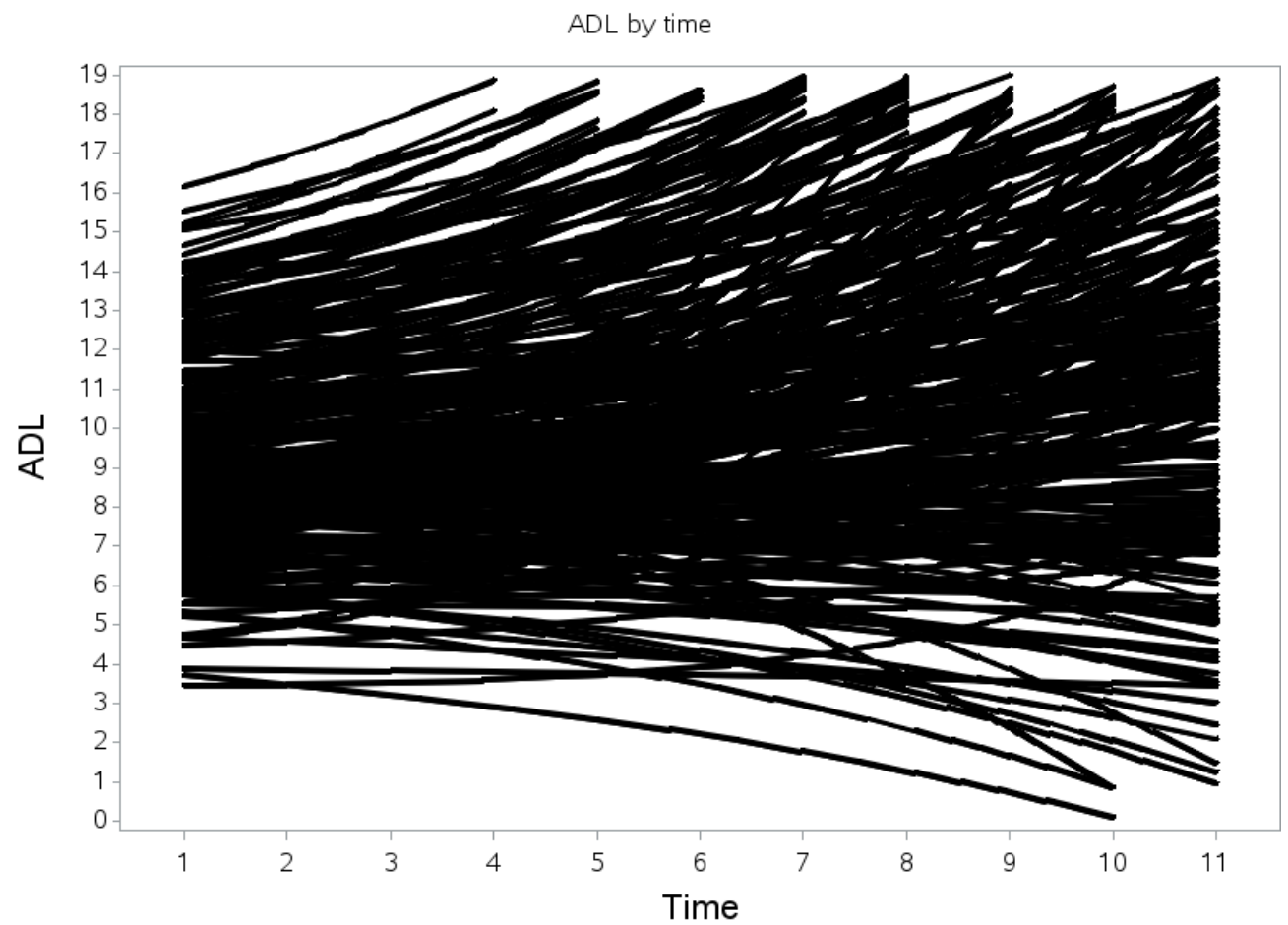

(b)

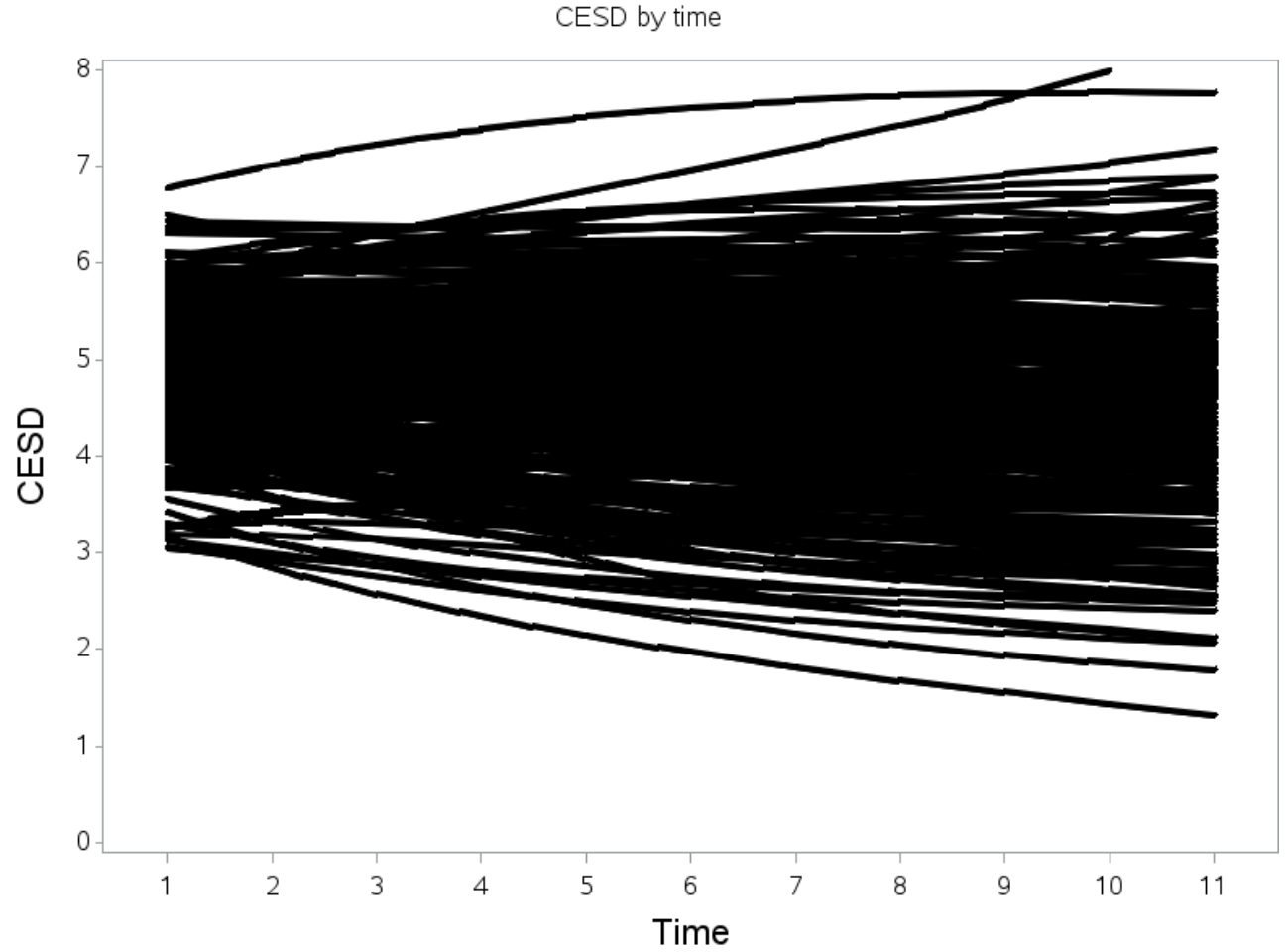


(c)

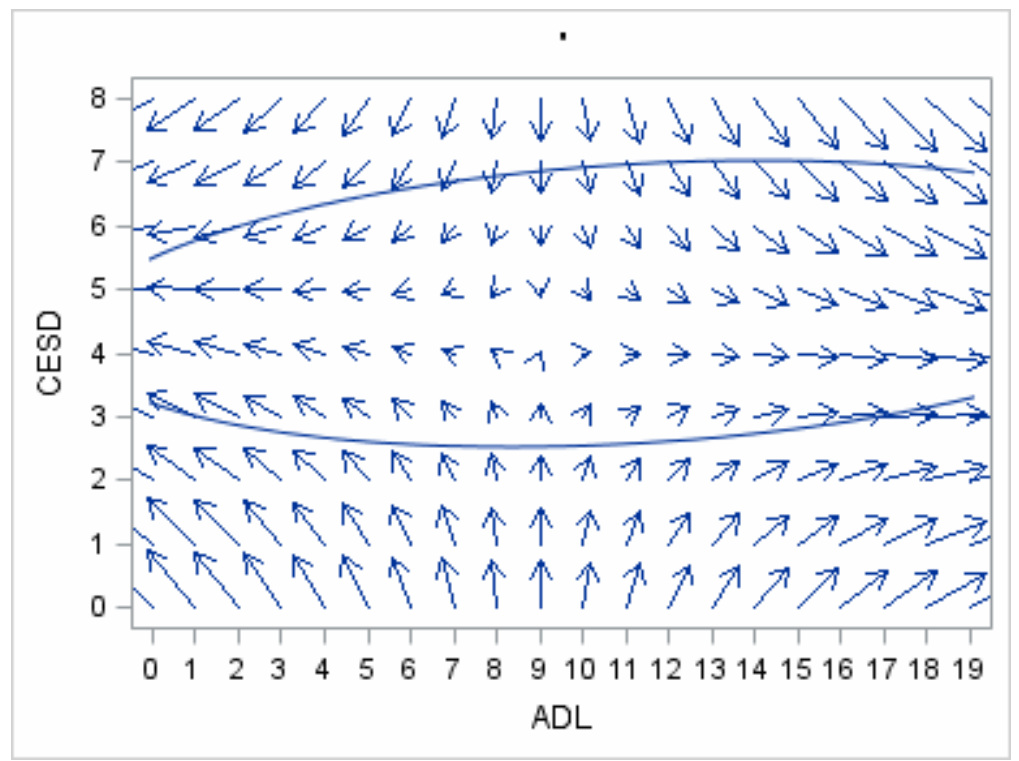

Figure 5. Plotting of change in ADLs and CESD over time and interaction between change in ADLs and CESD based on estimates from ADL-to-CESD bivariate dual change score model in latent class 1. (a) Plot of estimated ADL scores over time; (b) Plot of estimated CESD scores over time; (c) Vector plot of direction of change in ADL and CESD scores at different levels of each variable. 
(a)

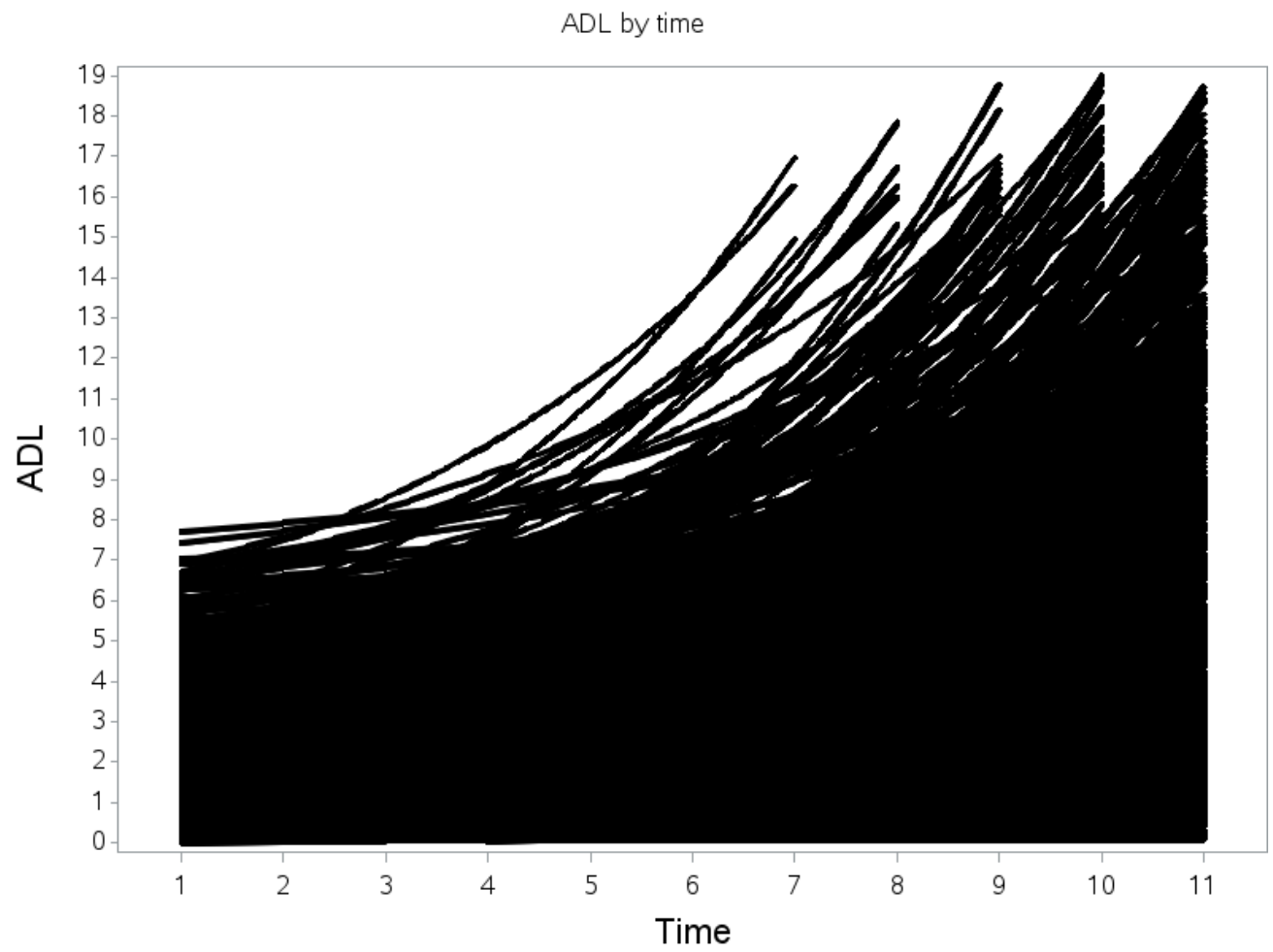

(b)

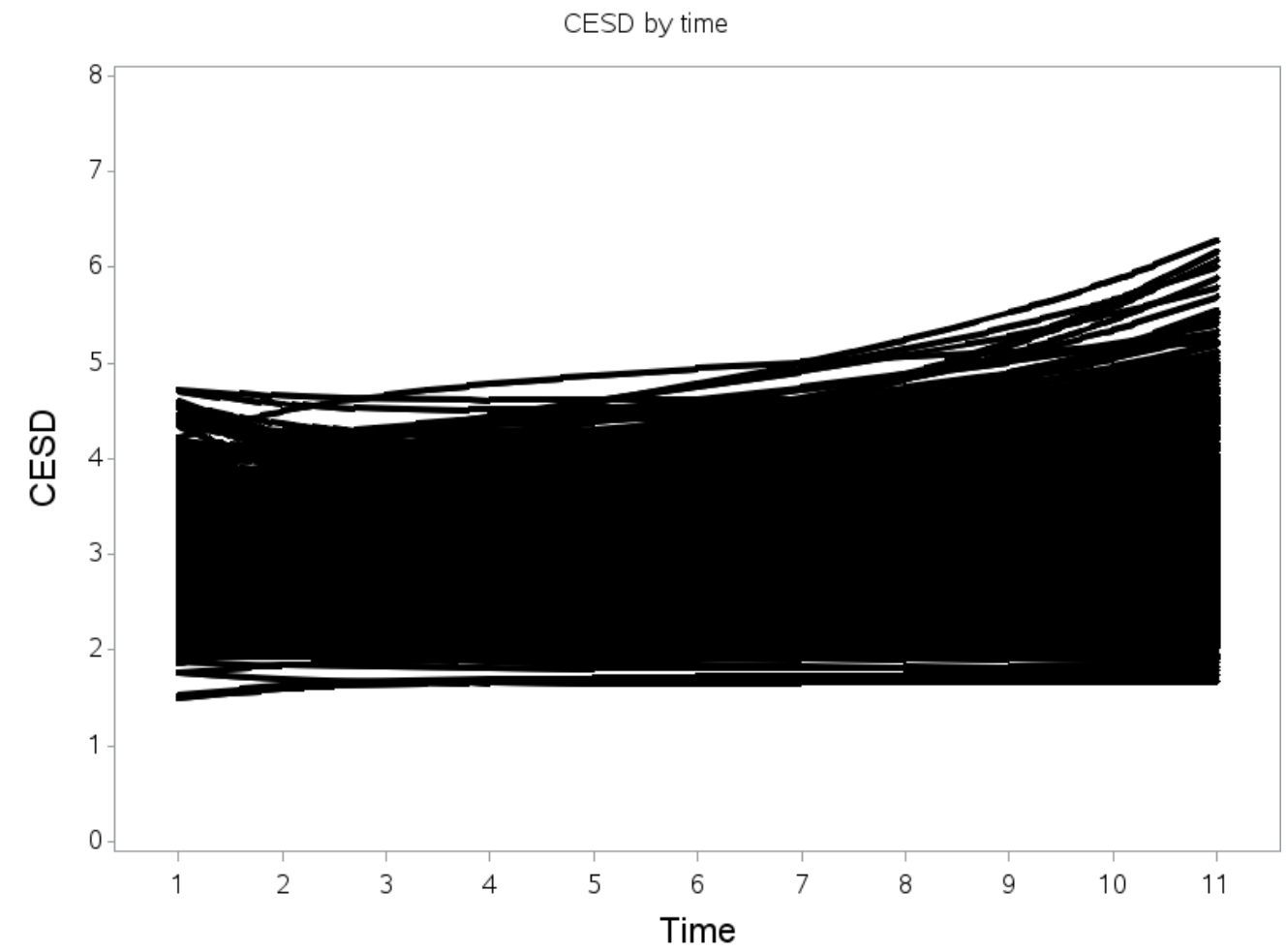


(c)

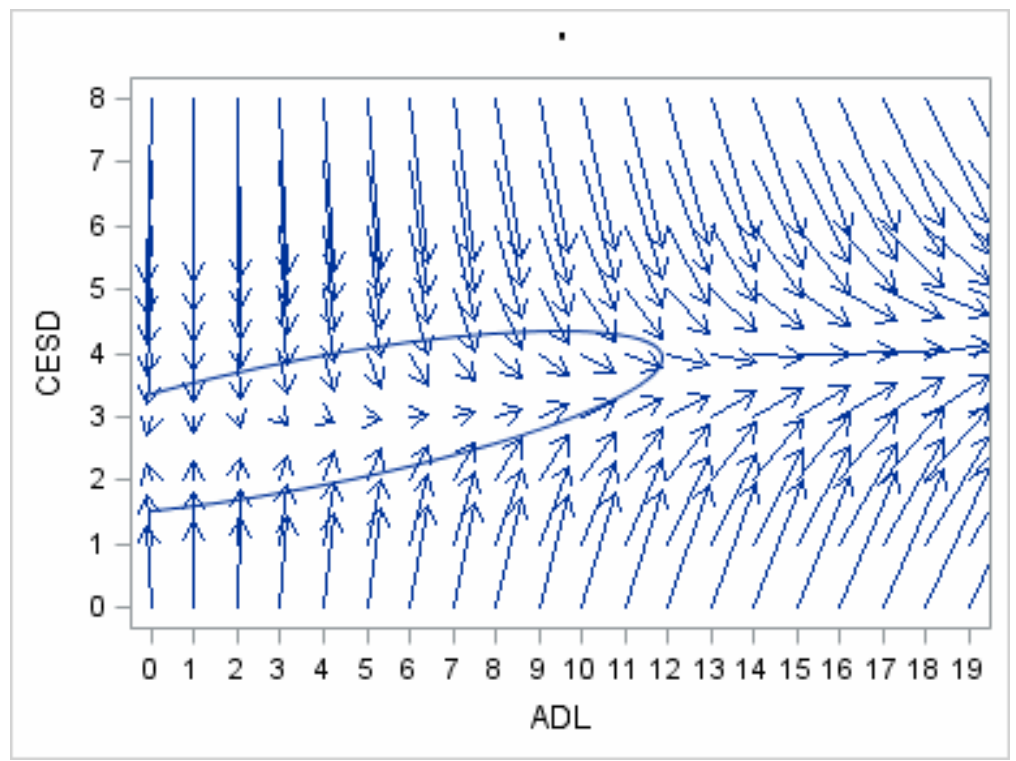

Figure 6. Plotting of change in ADLs and CESD over time and interaction between change in ADLs and CESD based on estimates from ADL-to-CESD bivariate dual change score model in latent class 2. (a) Plot of estimated ADL scores over time; (b) Plot of estimated CESD scores over time; (c) Vector plot of direction of change in ADL and CESD scores at different levels of each variable. 
(a)

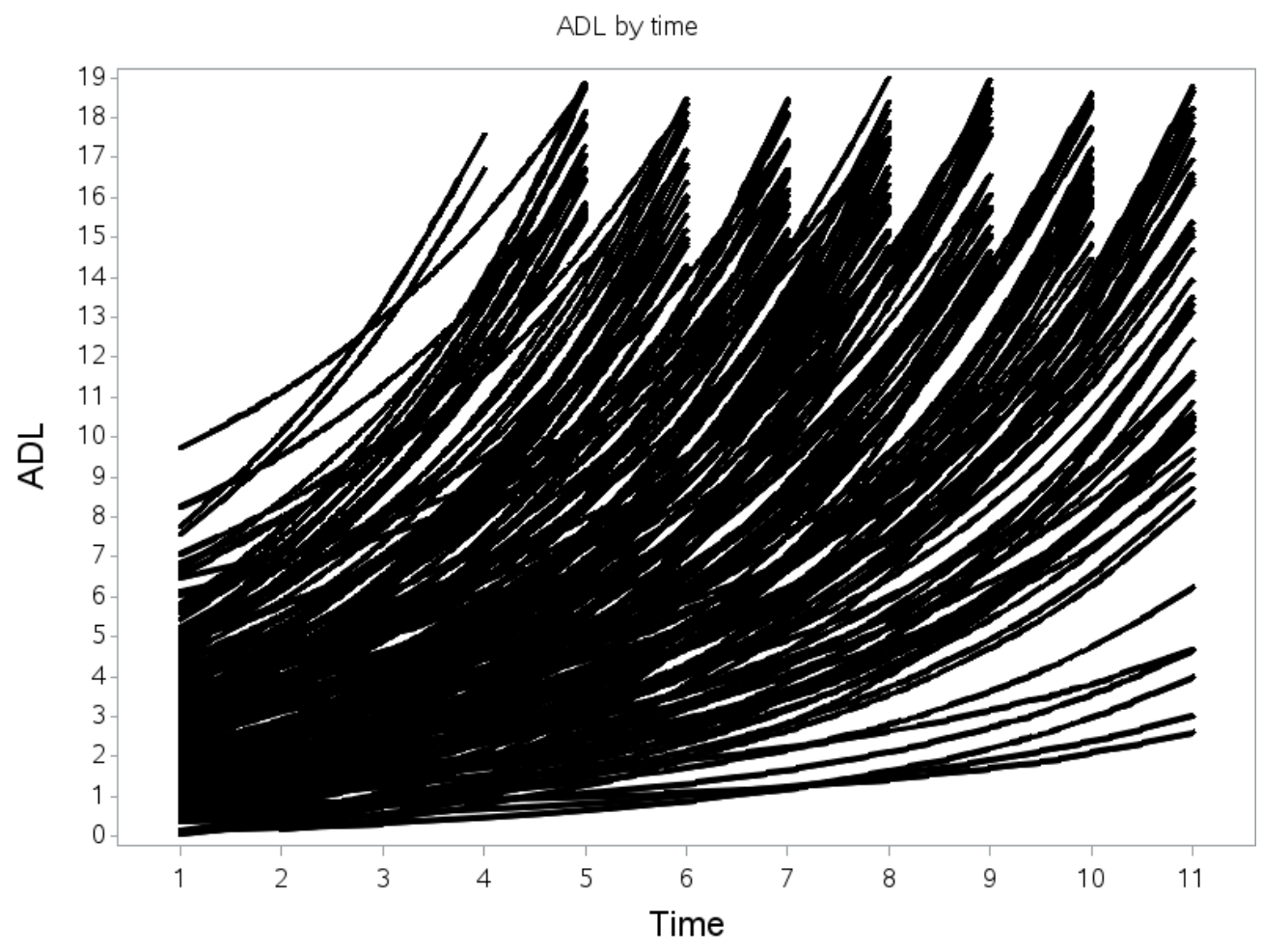

(b)

CESD by time

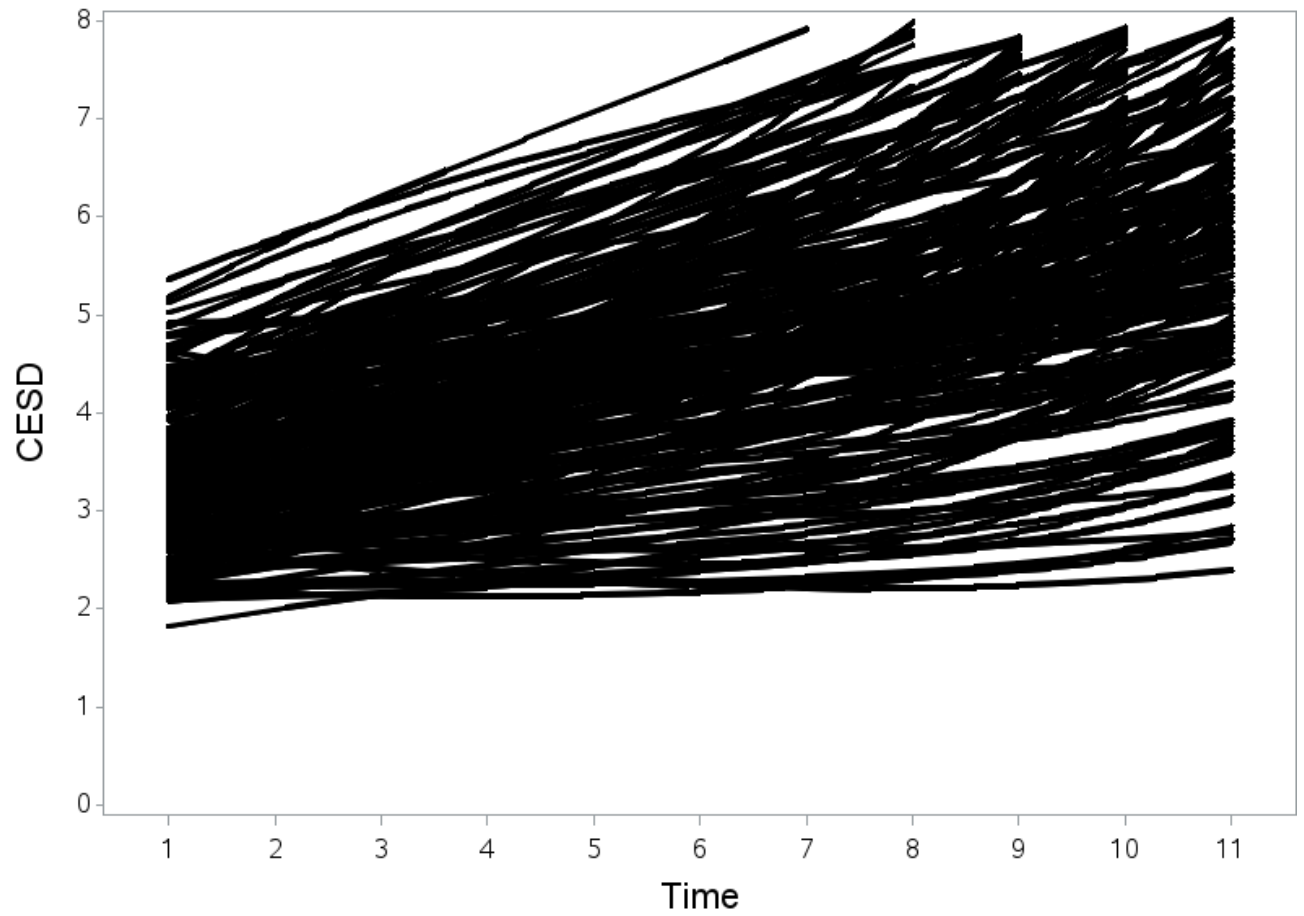


(c)

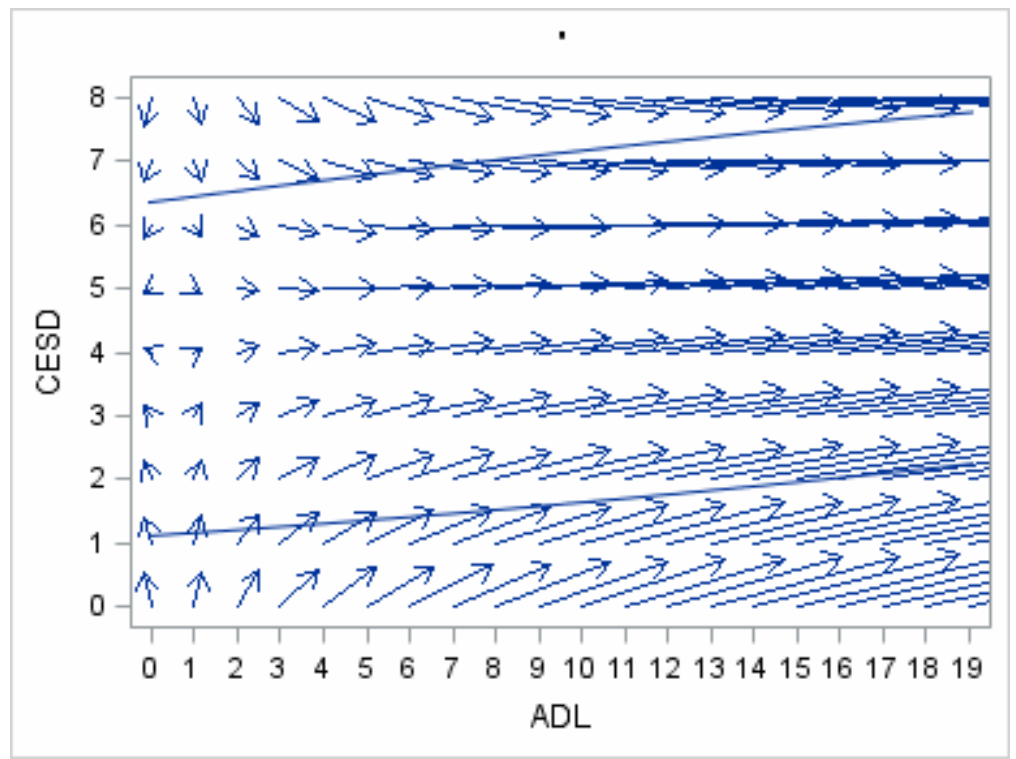

Figure 7. Plotting of change in ADLs and CESD over time and interaction between change in ADLs and CESD based on estimates from ADL-to-CESD bivariate dual change score model in latent class 3. (a) Plot of estimated ADL scores over time; (b) Plot of estimated CESD scores over time; (c) Vector plot of direction of change in ADL and CESD scores at different levels of each variable. 


\section{Appendix A}

Variables in the Health and Retirement Study

\section{Disability}

Scored: $1=$ yes (to difficulty), $5=$ no, $6=$ can't do, $7=$ don't do, $8=$ don't know, $9=$ refused

Recode: 1 or 6 recoded to $1 ; 5$ recoded to $0 ; 7,8$, and 9 recoded to missing - for sensitivity analyses, recoded 7 to 1 as well.

\section{Physical Activities}

1. Because of a health problem do you have any difficulty with walking several blocks? - If participant indicated they had no difficulty with this, they went on to \#2. If participant responded any other way, they went on to \#3.

2. ... with running or jogging about a mile? - If participant was administered this item, they then skipped to \#4.

3. ... with walking one block?

4. ... with sitting for about two hours?

5. ... with getting up from a chair after sitting for long periods?

6. ... with climbing several flights of stairs without resting? - If participant indicated they had no difficulty with this, they skipped to \#8. If participant responded any other way, they went on to \#7.

7. ... with climbing one flight of stairs without resting?

8. ... with stooping, kneeling, or crouching?

9. ... with reaching or extending your arms above shoulder level?

10.... with pulling or pushing large objects like a living room chair?

11... with lifting or carrying weights over 10 pounds, like a heavy bag of groceries? 
12. ... with picking up a dime from a table?

Activities of Daily Living - Administered only to participants who indicated having difficulty with at least one physical activity

1. Because of a health or memory problem do you have any difficulty with dressing, including putting on shoes and socks?

2. ... with walking across a room?

3. ... with bathing or showering?

4. ... with eating, such as cutting up your food?

5. ... with getting in or out of bed?

\section{Instrumental Activities of Daily Living}

1. Because of a health or memory problem, do you have any difficulty preparing a hot meal?

2. ... with shopping for groceries?

3. ... with making phone calls?

4. ... taking medications?

5. ... with managing you money - such as paying your bills and keeping track of expenses?

\section{Depressive Symptoms}

Scored: $1=$ yes, $5=$ no, $8=$ don't know, $9=$ refused

Recode: 1 recoded to $1 ; 5$ recoded to $0 ; 8$ and 9 recoded to missing

1. Much of the time during the past week, you felt depressed. Would you say yes or no?

2. ... you felt that everything you did was an effort....

3. ... your sleep was restless. ... 
4. ... you were happy.... [recode 5 to 1 and 1 to 0$]$

5. ... you felt lonely. ...

6. ... you enjoyed life.... [recode 5 to 1 and 1 to 0$]$

7. ... you felt sad. ...

8. ... you could not get going. ...

\section{Cognitive Status}

Coverscreen Proxy (available in 1994, 2000-2010)

1. Designate type of interview.

$1=$ self

$2=$ proxy, spouse/partner is reporter, and living in same household

$3=$ proxy, non-spouse/partner is reporter

$4=$ proxy, spouse/partner is reporter, but does not live in same household

Recode: 1 recoded to $0 ; 2,3$, and 4 recoded to 1

2. Do you have reason to think that [participant] would have difficulty completing this interview because of cognitive limitations?

$1=$ no reason to think [participant $]$ has any cognitive limitations

$2=[$ Participant $]$ may have some cognitive limitations but could probably do the interview

$3=[$ Participant $]$ has cognitive limitations that prevent him/her from being interviewed 


\section{Memory Problems (available in 1998-2008)}

Scored: $1=$ yes, 5 = no, $8=$ don't know, $9=$ refused

Recode: 1 recoded to $1 ; 5$ recoded to $0 ; 8$ and 9 recoded to missing

1. Has a doctor told you that you have a memory-related disease?

\section{Dementia/Alzheimer's Disease Diagnosis (available in 2010)}

Scored: 1 and $3=$ yes/now has condition, 4 and $5=$ no/does not have condition, $8=$ don't know, $9=$ refused

Recode: 1 and 3 recoded to $1 ; 4$ and 5 recoded to $0 ; 8$ and 9 recoded to missing

1. If previous record indicated individual had Alzheimer's disease, asked 1a. If previous record indicated individual did not have Alzheimer's disease, asked $1 \mathrm{~b}$.

1a. Our records from your last interview show that a doctor had told you that you had Alzheimer's disease.

1b. Since we last talked with you, has a doctor told you that you have Alzheimer's disease?

2. If previous record indicated individual had dementia, asked 2a. If previous record indicated individual did not have dementia, asked $2 b$.

2a. Our records from your last interview show that a doctor had told you that you had dementia, senility or some other serious memory impairment.

2b. Since we last talked with you, has a doctor told you that you have dementia, senility or any other serious memory impairment?

Cognitive status coded such that a participant was designated as cognitively impaired $(=1)$ if either a) he/she had a proxy for the interview and the proxy indicated "[Participant] has cognitive 
limitations that prevent him/her from being interviewed," or b) response was 1 or 3 (yes/now has condition) to the Alzheimer's disease or dementia item. 


\section{Appendix B}

Variables in the Swedish Twin Registry

\section{Disability}

Coded: $1=$ receive help, $0=$ no, missing $=$ don't know or refuse

1. Do you receive any help, for example with...

1a. Buying or preparing food

1b. Housework

1c. Personal care (for example dressing, bathing)

1d. Handling your finances

1e. Remembering to take your medicine

1f. Other

\section{Depressive Symptoms}

Coded: $0=$ never or almost never, $1=$ seldom, $2=$ often, $3=$ always or almost always, missing $=$ refuse

Instructions: For each of the following statements, please tell me if you experienced the feeling during the past week, and if so, how often?

1. You did not feel like eating. Your appetite was poor.

2. You felt depressed.

3. You felt that everything you did was an effort.

4. Your sleep was restless.

5. You were happy. [reverse-coded]

6. You felt lonely. 
7. People were unfriendly.

8. You enjoyed life. [reverse-coded]

9. You felt sad.

10. You felt that people disliked you.

11. You could not "get going." 